\title{
Joint Use of Scattered and Received Wave Polarization Information for Target Characterization and Scattering Power Decomposition
}

\author{
Subhadip Dey, Student Member, IEEE, Debanshu Ratha, Student Member, IEEE, \\ Dipankar Mandal, Student Member, IEEE, Avik Bhattacharya, Senior Member, IEEE, and \\ Alejandro C. Frery, Senior Member, IEEE,
}

\begin{abstract}
Incoherent target decomposition techniques provide unique scattering information from polarimetric SAR data either by fitting appropriate scattering models or by optimizing the "received" wave intensity through the diagonalization of the coherency (or covariance) matrix. Hence, the information provided by the "scattered" wave might be neglected. This scattered wave information can be well utilized to gain complete polarimetric information for numerous applications. In this study, a new roll-invariant scattering-type parameter is introduced, which jointly uses the degree of polarization as the "scattered" wave information and the elements of the covariance matrix as the "received" wave information from both full-polarimetric (FP) and compact-polarimetric (CP) SAR data. This scattering-type parameter, which is comparable to that of the Cloude $\alpha$ for FP SAR data and the ellipticity parameter $\chi$ for CP SAR data, can be well utilized to characterize various targets. Furthermore, this new scatteringtype parameter is adequately utilized to obtain a non-model based three-component scattering power decomposition technique. The double-bounce and the odd-bounce scattering powers are obtained by modulating the total polarized power by a proper geometrical factor easily derived using the new scattering-type parameter for both FP and CP SAR data. Moreover, due to its natural and direct formulation, the decomposition scattering powers are non-negative and roll-invariant while the total power is conserved. The proposed method is qualitatively and quantitatively assessed utilizing the L-band ALOS-2 and C-band Radarsat-2 FP and the associated simulated CP SAR data.
\end{abstract}

\section{Index Terms}

Full polarimetry, Compact polarimetry, Target decomposition, scattering type parameter, Target characterization

\section{INTRODUCTION}

$\mathbf{P}$ OLARIMETRIC DECOMPOSITIONS aim, among other applications, to characterize scattering mechanisms from a target. Broadly categorizing, target decomposition techniques are divided into two distinct categories: (1) coherent, and (2) incoherent. Coherent decomposition techniques utilize information from the $2 \times 2$ complex scattering matrix $[\mathbf{S}]$, whereas incoherent decompositions extract information from the second-order statistics in terms of the coherency $[\mathbf{T}]$ or the covariance $[\mathbf{C}] 3 \times 3$ matrices.

Several methods have been developed to decompose the average covariance or coherency matrices. In this regard, eigenvalue/eigenvector based decomposition methods provide a unique solution to the scattering mechanisms [1], [2]. The interpretation of the scattering information is achieved by obtaining a set of unique roll-invariant parameters. On the other hand, model-based decomposition methods utilize the target geometric and electromagnetic scattering properties to extract scattering mechanisms from the second-order statistics.

The pioneering work of Freeman and Durden [3] on the three-component scattering power decomposition (F3D) paved the way for model-based decomposition techniques. The scattering powers obtained from

S. Dey, D. Ratha, D. Mandal and A. Bhattacharya are with the Microwave Remote Sensing Lab, Center of Studies in Resources Engineering, Indian Institute of Technology Bombay, India, e-mail: (sdey2307@gmail.com).

Alejandro C. Frery is with the Universidade Federal de Alagoas, Maceió, Brazil, and the Key Lab of Intelligent Perception and Image Understanding of the Ministry of Education, Xidian University, Xi'an, China. (e-mail: acfrery@laccan.ufal.br). 
their method were based on the assumption of target reflection symmetry, i.e., $\left\langle S_{\mathrm{HH}} S_{\mathrm{HV}}^{*}\right\rangle=\left\langle S_{\mathrm{VV}} S_{\mathrm{VH}}^{*}\right\rangle=0$. The Freeman and Durden decomposition model is simple and easy to implement and has been utilized for several applications as, for instance, unsupervised classification [4], [5].

However, the reflection symmetry assumption is seldom verified for most of the targets in a typical scenario. Therefore, the condition of uncorrelated co-polarized and cross-polarized components does not hold, i.e., $\left\langle S_{\mathrm{HH}} S_{\mathrm{HV}}^{*}\right\rangle \neq 0$, and $\left\langle S_{\mathrm{VV}} S_{\mathrm{HV}}^{*}\right\rangle \neq 0$. In such a condition, the cross-polarized component, $\left\langle\left|S_{\mathrm{HV}}\right|^{2}\right\rangle$, might be predominant.

In this regard, Yamaguchi et al. proposed a four-component model-based decomposition technique [6], which incorporates a helix as a fourth component. In these two decompositions, the primary scatterer from vegetation canopy is modeled as a thin cylinder. However, such a description is often too simplistic for most vegetation complex structural configuration.

Hence, Arii et al. [7] proposed an $n^{\text {th }}$ power of cosine squared function to describe the complex canopy structure of vegetation. Nonetheless, this study considered the canopy scattering as the dominant mechanism. Similarly, Neumann et al. [8] proposed the retrieval of forest parameters using polarimetric interferometric SAR data. This study combined the physical model-based decomposition with a random volume over the ground inversion approach.

van Zyl et al. [9] proposed constraints to grant non-negative eigenvalues. Similarly, Cui et al. [10] performed the complete decomposition of the coherency matrix into one volume component and two single scattering components using a non-negative power constraint. Hence, this decomposition technique was able to overcome the negative power problem.

The aforementioned model-based decompositions did not take into account the target orientation aspect with the radar line of sight. Within a radar resolution cell, the targets can be randomly oriented about the radar line of sight and, thus, have diverse polarimetric responses. Several studies attempted to compensate this target orientation effect [11]-[13]. The central idea behind orientation compensation is to reduce the contribution of the cross-polarized component. In other words, the target orientation compensation minimizes the overestimation of the volume scattering power while increasing the double bounce scattering power.

Among these methods, Lee et al. [12], the three-component model-based decomposition (An3D) by An et al. [14], the four-component decomposition with the rotation of the coherency matrix by Yamaguchi et al. [15] are notable. Later, Bhattacharya et al. [16] utilized the degree of polarization of the scattered wave as an adaptive parameter to improve the scattering power components of the Yamaguchi four-component decomposition.

An alternative approach for determining the orientation of target while improving the scattering powers is Statistical Information Theory. In this regard, Bhattacharya et al. [17] optimized the Hellinger distance between orthogonal and rotated urban targets to the radar line of sight to determine the orientation angle for the modification of the Yamaguchi four-component decomposition powers. Subsequently, Eltoft et al. [18] extended the model-based decomposition techniques by introducing higher-order distribution function and radar texture models. An et al. [19] reconsidered the problem of negative scattering powers and the overestimation of the volume scattering component in Freeman-Durden decomposition. Here a methodology is proposed to completely decompose an arbitrary coherency matrix into several polarimetric symmetry components. Shuang et al. [20] combined a new condition with the Freeman-Durden decomposition to distinguish human-made structure and nature media after orientation angle compensation.

Full polarimetric (FP) SAR data provides optimum performance in target characterization due to the complete radar target information content. However, compact polarimetric SAR data offers more information than a single or dual-polarized SAR data, while covering larger swath widths compared to FP SAR systems.

In Compact Polarimetric (CP) radars, the relative phase between the two received polarizations is retained, unlike the conventional dual-polarized SAR systems. In the $\pi / 4$ mode [21], the transmitted polarization is a superposition of the linear horizontal $(\mathrm{H})$ and vertical polarization $(\mathrm{V})$ oriented at $45^{\circ}$ to the horizontal. The dual-circular compact polarimetry (DCP) mode proposed in [22] used right circular 
polarization on transmit and right and left circular polarization on receive. Furthermore, Raney [23] proposed a new hybrid-polarity architecture, consisting of circular transmit and orthogonal linear polarizations receive. This new hybrid-pol architecture preserves all the information of the DCP mode since the Stokes parameter of electromagnetic (EM) wave does not depend on the basis of the received polarization [24].

Raney et al. proposed the $m-\delta$ [23] and $m-\chi$ [25] decomposition methods for the hybrid-compact polarimetric SAR data, where $m$ indicates the degree of polarization of the scattered EM wave. The performance of the $m-\delta$ decomposition profoundly depends on the purity in the transmission polarization of the EM wave. Hence, the phase difference parameter, $\delta$, provides better results only when the transmitted wave is perfectly circular. On the contrary, the ellipticity, $\chi$, is robust towards the transmitting wave polarization. While characterizing the scattering phenomenon from the lunar surface, Raney et al. [25] pointed out the ability of the $m-\chi$ decomposition to resolve certain even-odd bounce scattering ambiguity over lunar crater walls. Moreover, the authors proposed a three-component $(m-\chi-\psi)$ decomposition, more appropriate to discriminate different scattering mechanisms with a priori information of the transmitting ellipticity $(\chi)$ of the EM wave. This striking idea was exploited by Bhattacharya et al. [26] while proposing the $S$ - $\Omega$ decomposition, where $\Omega$ depends on $m$, the transmitting wave ellipticity $\left(\chi_{t}\right)$ and orientation $\left(\psi_{t}\right)$ and the received wave ellipticity $\left(\chi_{r}\right)$ and orientation $\left(\psi_{r}\right)$.

Incoherent target decomposition techniques might not utilize complete polarimetric information present in SAR data. In particular, such techniques for full polarimetric SAR data optimize the received wave intensity through the diagonalization of the coherency (or covariance) matrix [2], [27], [28]. Hence, the information provided by the scattered wave in terms of the degree of polarization might be ignored. This scattered wave parameter can be well utilized to recover the polarimetric information omitted by the received wave polarization [29].

In this study, we use the scattered wave information in terms of the degree of polarization $(m)$ and the received wave intensities from the coherency (or covariance) matrix to obtain a roll-invariant scatteringtype parameter for both FP and CP SAR data. The role of this parameter is comparable to the Cloude and Pottier $\alpha$ [1] for FP, and the wave ellipticity $(\chi)$ for CP SAR data. Furthermore, we propose a novel threecomponent scattering power decomposition technique for both FP and CP SAR data by jointly utilizing the scattering-type parameter and the degree of polarization.

Unlike traditional model-based decompositions, the proposed method does not utilize any particular scattering models for the estimation of the scattering powers. Moreover, each power component is guaranteed to be non-negative, and the total power is conserved. The proposed "non-model" three-component scattering power decomposition technique is applied to full and simulated hybrid-compact polarimetric Lband ALOS-2 and C-band RS-2 SAR data over Mumbai and San Francisco, respectively. The effectiveness of the proposed method is used for qualitative analysis of scattering mechanisms and quantitative analysis of the scattering powers.

This work unfolds as follows. We derived a new target characterization parameter for full and compact polarimetric SAR data in Section II, which is further utilized to obtain a unique three-component nonmodel based scattering power decomposition for two datasets. In Section III, we compare the results obtained from the proposed technique with other existing target characterization parameters and decomposition techniques. Section III-A1 and section III-B1 compare the proposed target characterization parameters with the ones existing in literature for FP and CP data. Accordingly, section III-A2 and section III-B2 present and compare the proposed 3-component non-model based decomposed powers with two decomposition techniques for FP and CP SAR data respectively. Finally, Section IV summarizes the proposed methodology and concludes by highlighting its advantages and limitations for different SAR data.

\section{Methodology}

A new roll-invariant scattering-type parameter is introduced, which utilizes the degree of polarization $(m)$ as a scattered wave information and the elements of the covariance matrix for FP and CP SAR 
data as the received wave information. In this regard, an expression derived from [30] is utilized for calculating the degree of polarization from pixels. This scattering-type parameter is then effectively used to obtain a non-model based three-component scattering power decomposition. The double-bounce and the odd-bounce powers are obtained by modulating the total polarized power by a precise geometrical factor easily derived using the new scattering-type parameter for both FP and CP SAR data.

\section{A. Full Polarimetry}

In FP SAR, the $2 \times 2$ complex scattering matrix $\mathbf{S}$ encompasses complete polarimetric information about backscattering from targets for each pixel. It is expressed in the backscatter alignment (BSA) convention in the linear horizontal $(\mathrm{H})$ and linear vertical $(\mathrm{V})$ polarization basis as,

$$
\mathbf{S}=\left[\begin{array}{ll}
S_{\mathrm{HH}} & S_{\mathrm{HV}} \\
S_{\mathrm{VH}} & S_{\mathrm{VV}}
\end{array}\right] \Rightarrow \boldsymbol{k}=V([\mathbf{S}])=\frac{1}{2} \operatorname{Tr}(\mathbf{S} \Psi)
$$

where $V(\cdot)$ is the vectorization operator on the scattering matrix, $\Psi$ is the corresponding basis matrix, and $\operatorname{Tr}$ is the sum of the diagonal elements of the matrix. Each element of the matrix represents the backscattering response of the target at a specific polarization. The diagonal elements of the matrix represent the co-polarized scattering information, while the off-diagonal terms represent the cross-polarized information. In the monostatic backscattering case, the reciprocity theorem constrains the scattering matrix to be symmetric, i.e., $S_{\mathrm{HV}}=S_{\mathrm{VH}}$.

The multi-looked Hermitian positive semi-definite $3 \times 3$ coherency matrix $\mathbf{T}$ is obtained from the averaged outer product of the target vector $\boldsymbol{k}_{P}$ (derived using the Pauli basis matrix, $\Psi_{P}$ ) with its conjugate. Similarly, the $3 \times 3$ covariance matrix $\mathbf{C}$ is obtained from the averaged outer product of the target vector $\boldsymbol{k}_{L}$ (derived using the Lexicographic basis matrix, $\Psi_{L}$ ) with its conjugate.

$$
\begin{aligned}
& \Psi_{P}=\left\{\sqrt{2}\left[\begin{array}{ll}
1 & 0 \\
0 & 1
\end{array}\right], \sqrt{2}\left[\begin{array}{cc}
1 & 0 \\
0 & -1
\end{array}\right], \sqrt{2}\left[\begin{array}{ll}
0 & 1 \\
1 & 0
\end{array}\right]\right\} \\
& \Psi_{L}=\left\{2\left[\begin{array}{ll}
1 & 0 \\
0 & 0
\end{array}\right], 2 \sqrt{2}\left[\begin{array}{ll}
0 & 1 \\
0 & 0
\end{array}\right], 2\left[\begin{array}{ll}
0 & 0 \\
0 & 1
\end{array}\right]\right\}
\end{aligned}
$$

The state of polarization of an EM wave is characterized in terms of the degree of polarization $(0 \leq$ $m \leq 1)$. The degree of polarization is defined as the ratio of the (average) intensity of the polarized portion of the wave to that of the (average) total intensity of the wave. For a completely polarized EM wave, $m=1$ and for a completely unpolarized EM wave, $m=0$. In between these two extreme cases, the EM wave is said to be partially polarized, $0<m<1$.

Barakat [30] provided an expression of $m$ for the $N \times N$ coherency matrix. This expression is used in this study to obtain the degree of polarization $m_{\mathrm{FP}}$ from the $3 \times 3$ coherency matrix $\mathbf{T}$ for FP SAR data as,

$$
m_{\mathrm{FP}}=\sqrt{1-\frac{27|\mathbf{T}|}{(\operatorname{Tr}(\mathbf{T}))^{3}}}
$$

where $|\cdot|$ is the determinant of a matrix.

Without any loss of generality, let us assume,

$$
\tan \alpha_{1}=\frac{T_{11}}{m_{\mathrm{FP}} \cdot \operatorname{Span}} \quad \text { and } \quad \tan \psi_{1}=\frac{T_{22}+T_{33}}{m_{\mathrm{FP}} \cdot \operatorname{Span}},
$$

where $T_{11}, T_{22}$, and $T_{33}$ are the diagonal elements of the coherency matrix and,

$$
\text { Span }=T_{11}+T_{22}+T_{33}
$$


Therefore, by using a simple trigonometrical relationship, we get,

$$
\begin{aligned}
\tan \theta_{\mathrm{FP}} & =\tan \left(\alpha_{1}-\psi_{1}\right) \\
& =\frac{m_{\mathrm{FP}} \cdot \operatorname{Span} \cdot\left(T_{11}-T_{22}-T_{33}\right)}{T_{11} \cdot\left(T_{22}+T_{33}\right)+m_{\mathrm{FP}}^{2} \cdot \mathrm{Span}^{2}}
\end{aligned}
$$

and following this,

$$
\sin 2 \theta_{\mathrm{FP}}=\tan \theta_{\mathrm{FP}}\left(1+\cos 2 \theta_{\mathrm{FP}}\right) .
$$

It can be observed that $\theta_{\mathrm{FP}} \in\left[-\frac{\pi}{4}, \frac{\pi}{4}\right]$ is a roll-invariant parameter which can be used to characterize scattering type information from targets.

From (5), when $m_{\mathrm{FP}}=0$ (i.e. when no polarization structure exists in the scattered EM wave) then $\theta_{\mathrm{FP}}=0$. Whereas, when $m_{\mathrm{FP}}=1$, either $\theta_{\mathrm{FP}}=-\frac{\pi}{4}$ or $\theta_{\mathrm{FP}}=\frac{\pi}{4}$, depending on the scattering from a dihedral or a trihedral target respectively. Otherwise, for all other cases, $\theta_{\mathrm{FP}} \in\left(-\frac{\pi}{4}, \frac{\pi}{4}\right)$.

The degree of polarization, $m_{\mathrm{FP}}$, and the scattering type information, $\theta_{\mathrm{FP}}$ is used to split the total power (Span) into two components: double bounce $\left(P_{d}^{\mathrm{FP}}\right)$ and surface $\left(P_{s}^{\mathrm{FP}}\right)$ scattering powers using a geometrical factor $\left(1 \pm \sin 2 \theta_{\mathrm{FP}}\right)$. The volume scattering power $\left(P_{v}^{\mathrm{FP}}\right)$ is then obtained as the depolarized fraction of the total power,

$$
\begin{aligned}
P_{d}^{\mathrm{FP}} & =\frac{m_{\mathrm{FP}} \cdot \operatorname{Span}}{2}\left(1-\sin 2 \theta_{\mathrm{FP}}\right), \text { and } \\
P_{v}^{\mathrm{FP}} & =\operatorname{Span} \cdot\left(1-m_{\mathrm{FP}}\right) \\
P_{s}^{\mathrm{FP}} & =\frac{m_{\mathrm{FP}} \cdot \operatorname{Span}}{2}\left(1+\sin 2 \theta_{\mathrm{FP}}\right),
\end{aligned}
$$

In the case of scattering power decomposition, when $m_{\mathrm{FP}}=0, P_{d}^{\mathrm{FP}}=P_{s}^{\mathrm{FP}}=0$, and $P_{v}^{\mathrm{FP}}=$ Span. This situation corresponds to the complete depolarized case. For pure even bounce scattering, $m_{\mathrm{FP}}=1$ and $\theta_{\mathrm{FP}}=-\frac{\pi}{4}$ with $P_{s}^{\mathrm{FP}}=P_{v}^{\mathrm{FP}}=0$, and $P_{d}^{\mathrm{FP}}=$ Span. For pure odd bounce scattering, $m_{\mathrm{FP}}=1$ and $\theta_{\mathrm{FP}}=\frac{\pi}{4}$ with $P_{d}^{\mathrm{FP}}=P_{v}^{\mathrm{FP}}=0$, and $P_{s}^{\mathrm{FP}}=$ Span. It is noteworthy that each scattering power component is non-negative, and the total power (Span) is conserved for any polarization state.

\section{B. Compact Polarimetry} $\mathrm{S}$ as,

The hybrid compact polarimetric mode measures a projection of the $2 \times 2$ complex scattering matrix

$$
\begin{aligned}
{\left[\begin{array}{c}
E_{C \mathrm{H}} \\
E_{C \mathrm{~V}}
\end{array}\right] } & =\frac{1}{\sqrt{2}}\left[\begin{array}{ll}
S_{\mathrm{HH}} & S_{\mathrm{HV}} \\
S_{\mathrm{VH}} & S_{\mathrm{VV}}
\end{array}\right]\left[\begin{array}{c}
1 \\
\pm i
\end{array}\right] \\
& =\frac{1}{\sqrt{2}}\left[\begin{array}{c}
S_{\mathrm{HH}} \pm i S_{\mathrm{HV}} \\
S_{\mathrm{VH}} \pm i S_{\mathrm{VV}}
\end{array}\right]
\end{aligned}
$$

where the subscript $C$ can be either the left-hand circular (LHC) transmit with a + sign or the right-hand circular (RHC) transmit with a - sign. The $2 \times 2$ covariance matrix $\mathbf{C}_{2}$ is then obtained from the elements of the scattering vector as,

$$
\mathbf{C}_{2}=\left[\begin{array}{cc}
\left\langle\left|E_{C \mathrm{H}}\right|^{2}\right\rangle & \left\langle E_{C \mathrm{H}} E_{C \mathrm{~V}}^{*}\right\rangle \\
\left\langle E_{C \mathrm{~V}} E_{C \mathrm{H}}^{*}\right\rangle & \left\langle\left|E_{C \mathrm{~V}}\right|^{2}\right\rangle
\end{array}\right] .
$$

For CP-SAR data, the $4 \times 1$ Stokes vector $\overrightarrow{\mathbf{S}}$ can be written in terms of the elements of the $2 \times 2$ covariance matrix $\mathrm{C}_{2}$ as,

$$
\overrightarrow{\mathbf{S}}=\left[\begin{array}{c}
S_{0} \\
S_{1} \\
S_{2} \\
S_{3}
\end{array}\right]=\left[\begin{array}{c}
C_{11}+C_{22} \\
C_{11}-C_{22} \\
C_{12}+C_{21} \\
\pm j\left(C_{12}-C_{21}\right)
\end{array}\right]
$$


where \pm corresponds to left and right circular polarization respectively.

The first element of the Stokes vector $S_{0}$ is a measure of the total average received power, and the third element $S_{3}$ is a measure of the average received power in circular polarization. The handedness of this circular polarization can be inferred by the sign $( \pm)$ of the $S_{3}$ component.

The proportion of the power that is received by the radar in opposite-sense circular polarization to that transmitted is $\left(S_{0}+S_{3}\right) / 2$. For example, $\mathrm{OC}=\left(S_{0}+S_{3}\right) / 2=\left|E_{\mathrm{R}}\right|^{2}$ for left-circular (L) polarization on transmit, where $\left|E_{\mathrm{R}}\right|^{2}$ is the intensity of the right-circular component. Such a change of polarity occurs when an EM wave undergoes an odd number of reflections from a target.

Similarly, $\left(S_{0}-S_{3}\right) / 2$ is a measure of the power received by the radar in the same-sense circular polarization as it was transmitted, which represents an even number of reflections from a target. For example, $\mathrm{SC}=\left(S_{0}-S_{3}\right) / 2=\left|E_{\mathrm{L}}\right|^{2}$ for left-circular (L) polarization on transmit, where $\left|E_{\mathrm{L}}\right|^{2}$ is the intensity of the left-circular component.

Similarly to the FP case, we use the Barakat formulation to obtain the degree of polarization $m_{\mathrm{CP}}$ from the $2 \times 2$ covariance matrix $\mathrm{C}_{2}$ for $\mathrm{CP}$ SAR data as,

$$
m_{\mathrm{CP}}=\sqrt{1-\frac{4\left|\mathbf{C}_{2}\right|}{\left(\operatorname{Tr}\left(\mathbf{C}_{2}\right)\right)^{2}}} .
$$

Similar to the FP case, without any loss of generality, let us assume,

$$
\tan \alpha_{2}=\frac{\mathrm{OC}}{m_{\mathrm{CP}} \cdot S_{0}} \quad \text { and } \quad \tan \psi_{2}=\frac{\mathrm{SC}}{m_{\mathrm{CP}} \cdot S_{0}},
$$

with,

$$
S_{0}=\mathrm{SC}+\mathrm{OC}
$$

Similarly, by using a simple trigonometrical relationship, we obtain,

$$
\begin{aligned}
\tan \theta_{\mathrm{CP}} & =\tan \left(\alpha_{2}-\psi_{2}\right) \\
& =\frac{m_{\mathrm{CP}} \cdot S_{0} \cdot(\mathrm{OC}-\mathrm{SC})}{\mathrm{OC} \cdot \mathrm{SC}+m_{\mathrm{CP}}^{2} \cdot S_{0}^{2}}
\end{aligned}
$$

and

$$
\sin 2 \theta_{\mathrm{CP}}=\tan \theta_{\mathrm{CP}}\left(1+\cos 2 \theta_{\mathrm{CP}}\right) .
$$

Here, $\theta_{\mathrm{CP}} \in\left[-\frac{\pi}{4}, \frac{\pi}{4}\right]$ characterizes scattering type information from targets which uses the same sense (SC) and opposite sense (OC) scattered powers in its derivation.

It can be observed from equation (17) that when $m_{\mathrm{CP}}=0$ (i.e. when no polarization structure exists in the scattered EM wave) then $\theta_{\mathrm{CP}}=0$. Whereas, when $m_{\mathrm{CP}}=1$, either $\theta_{\mathrm{CP}}=-\frac{\pi}{4}$ or $\theta_{\mathrm{CP}}=\frac{\pi}{4}$ depending on the sense (i.e., right or left circular) of the received polarization with respect to the transmit polarization. Otherwise, for all other cases, $\theta_{\mathrm{CP}} \in\left(-\frac{\pi}{4}, \frac{\pi}{4}\right)$.

Analogously to the FP case, the degree of polarization, $m_{\mathrm{CP}}$, and the scattering type information, $\theta_{\mathrm{CP}}$ is used to split the total power (Span) into two components: double bounce $\left(P_{d}^{\mathrm{CP}}\right)$ and surface $\left(P_{s}^{\mathrm{CP}}\right)$ scattering powers using a geometrical factor $\left(1 \pm \sin 2 \theta_{\mathrm{CP}}\right)$. Similarly, the volume scattering power $\left(P_{v}^{\mathrm{CP}}\right)$ is then obtained as the depolarized fraction of the total power:

$$
\begin{aligned}
P_{d}^{\mathrm{CP}} & =\frac{m_{\mathrm{CP}} \cdot S_{0}}{2}\left(1-\sin 2 \theta_{\mathrm{CP}}\right), \text { and } \\
P_{v}^{\mathrm{CP}} & =S_{0} \cdot\left(1-m_{\mathrm{CP}}\right) . \\
P_{s}^{\mathrm{CP}} & =\frac{m_{\mathrm{CP}} \cdot S_{0}}{2}\left(1+\sin 2 \theta_{\mathrm{CP}}\right),
\end{aligned}
$$


In the case of scattering power decomposition, when $m_{\mathrm{CP}}=0, P_{d}^{\mathrm{CP}}=P_{s}^{\mathrm{CP}}=0$, and $P_{v}^{\mathrm{CP}}=S_{0}$. This corresponds to the complete depolarized case. For pure even bounce scattering, $m_{\mathrm{CP}}=1$ and $\theta_{\mathrm{CP}}=-\frac{\pi}{4}$ with $P_{s}^{\mathrm{CP}}=P_{v}^{\mathrm{CP}}=0$, and $P_{d}^{\mathrm{CP}}=S_{0}$. For pure odd bounce scattering, $m_{\mathrm{CP}}=1$ and $\theta_{\mathrm{CP}}=\frac{\pi}{4}$ with $P_{d}^{\mathrm{CP}}=P_{v}^{\mathrm{CP}}=0$, and $P_{s}^{\mathrm{CP}}=S_{0}$. It can be observed that the total scattering power is conserved $\left(S_{0}\right)$ for any polarization states.

\section{RESULTS}

In the previous section, we have derived two roll-invariant parameters, namely $\theta_{\mathrm{FP}}$ and $\theta_{\mathrm{CP}}$ by jointly utilizing the scattered and received wave information. These two parameters are obtained from different formulations due to two different imaging modes (i.e., FP and CP). Nevertheless, their physical interpretation is comparable to some of the traditional parameters known in the literature, viz., Cloude and Pottier's $\alpha$ [31] for FP, and Raney et al.'s $\chi$ [25] for CP SAR data. This section provides a comparison of $\theta_{\mathrm{FP}}$ and $\theta_{\mathrm{CP}}$ with $\bar{\alpha}=45^{\circ}-\alpha$ and $\bar{\chi}=-\chi$ respectively. The translations of $\alpha$ and $\chi$ to $\bar{\alpha}$ and $\bar{\chi}$, respectively, are given only to compare the scattering nature of the targets within the same range.

We used two full polarimetric (FP) SAR images over Mumbai, India, and San Francisco (SF), USA, shown in Fig. 1. The Mumbai scene is in L-band with a center incidence angle of $33^{\circ}$. The image is multilooked with a factor of 3 in range direction, and 5 in the azimuth direction to generate $15 \mathrm{~m}^{2}$ pixels.

The SF scene is a C-band RS-2 image acquired with near and far range incidence angles of $28.02^{\circ}$ and $29.81^{\circ}$, respectively. The single look complex (SLC) image is multilooked with a factor of 2 in the range direction, and 4 in the azimuth direction to generate a $20 \mathrm{~m}^{2}$ ground pixel.

Additionally, we generated simulated hybrid-compact polarimetric (CP) data from both the FP data with an ellipticity angle of $-45^{\circ}$ (right circular) and $0^{\circ}$ orientation angle.

\section{A. Full polarimetry}

Figs. 2 and 3 show the images of $\theta_{\mathrm{FP}}$ and $\bar{\alpha}$ for the ALOS-2 L-band and RS-2 C-band SAR data. It can be seen that the $\theta_{\mathrm{FP}}$ reveals more details over different land cover classes.

Fig. 4 shows transects that contain different land cover features over both FP images, and the values of the proposed parameter $\theta_{\mathrm{FP}}$ along with the available parameter $\bar{\alpha}=45^{\circ}-\alpha$ for comparison. Fig. $4 \mathrm{a}$ shows $\theta_{\mathrm{FP}}$ over a transect in the ALOS-2 L-band Mumbai image, while Fig. 4b shows its values over a transect in the Radarsat-2 C-band San-Francisco. In both cases, for every 10 values over the transects, the averaged value is plotted to produce a smooth appearance.

Fig. 5 shows histograms of $\theta_{\mathrm{FP}}$, along with their notched boxplots (the notches are approximate confidence intervals for the median at $95 \%$ ), over selected areas of both FP images, identified as "A" (urban), "B" (sea), and "C" (forest) in Fig. 1. Figures 5a and 5b show the histograms and boxplots of $\theta_{\mathrm{FP}}$ over these three regions for the two images.

1) Comparison of $\theta_{F P}$ with $\bar{\alpha}$ : Fig. 4a shows that both $\bar{\alpha}$ and $\theta_{\mathrm{FP}}$ follow a similar trend over the transect. Such a response indicates a comparable behavior at characterizing scattering types from different targets.

We highlight, however, notable differences in the boxes FM1, FM2, FM3, and FM4 over the Mumbai transect. FM1 and FM3 belong to the ocean surface, FM2 belongs to the urban area, and FM4 belongs to the vegetation area.

In FM1 and FM3, the degree of polarization $\left(m_{\mathrm{FP}}\right)$ is in between 0.95 and 1 , and both $\theta_{\mathrm{FP}}$ and $\bar{\alpha}$ are positive. In general, when the ocean surface is smooth, the co-polar signatures obtained from the ocean surface show a low coefficient of variation associated with a high degree of polarization [32]. Alongside, over the ocean surface, an odd bounce scattering mechanism dominates due to which both $\theta_{\mathrm{FP}}$ and $\bar{\alpha}$ reach positive values. However, the value of $\bar{\alpha}$ is roughly between $27^{\circ}$ to $29^{\circ}$, whereas the value of $\theta_{\mathrm{FP}}$ is around $35^{\circ}$ to $37^{\circ}$.

Similar to $m_{\mathrm{FP}}$ over FM1, the value of $m_{\mathrm{FP}}$ over FM2 is also high. However, the values of $\bar{\alpha}$ and $\theta_{\mathrm{FP}}$ lead to infer the presence of even bounce scattering from these urban areas. Besides, the value of $\theta_{\mathrm{FP}}$ is around $1^{\circ}$ to $2^{\circ}$ higher than $\bar{\alpha}$. On the other hand, the value of $m_{\mathrm{FP}}$ over FM4 is low, which might be due 


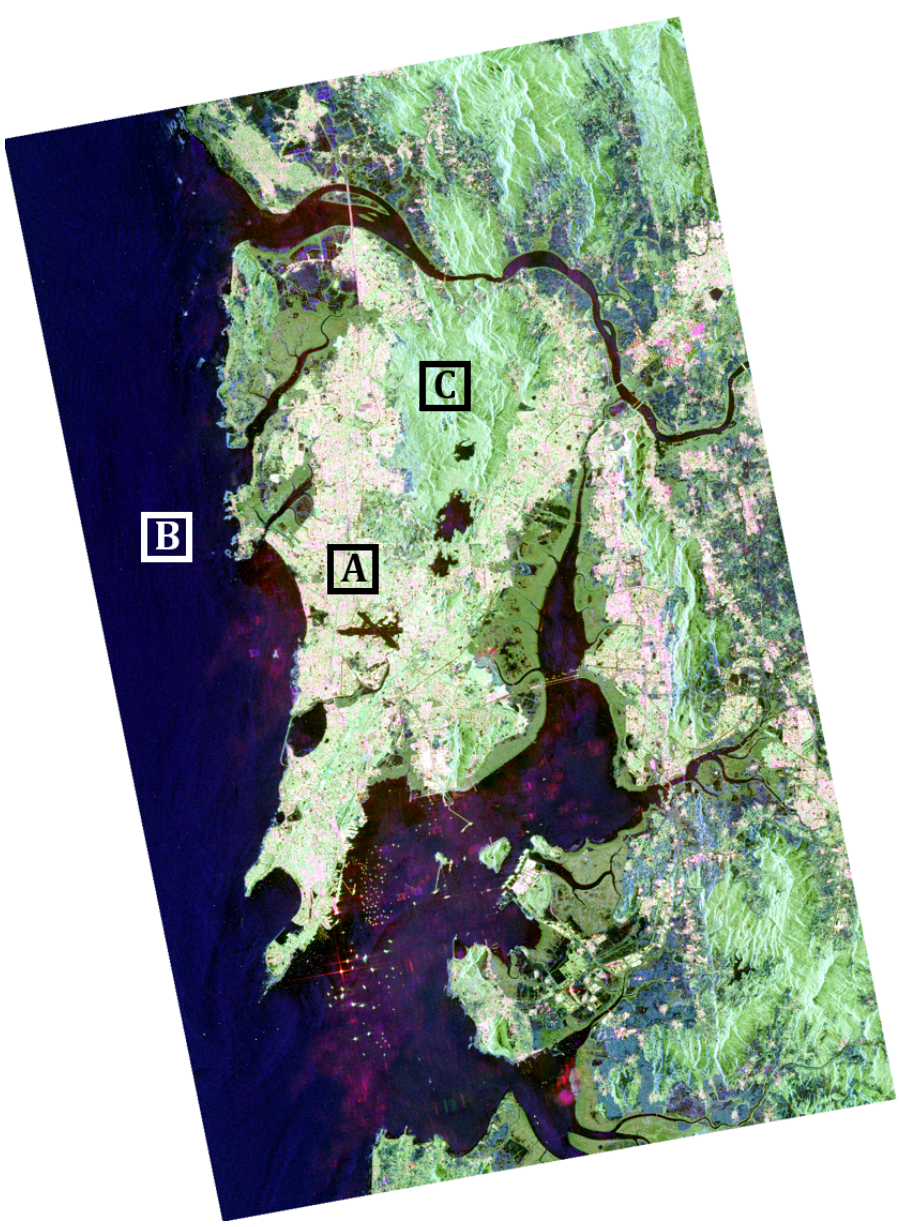

(a) Pauli RGB, Mumbai

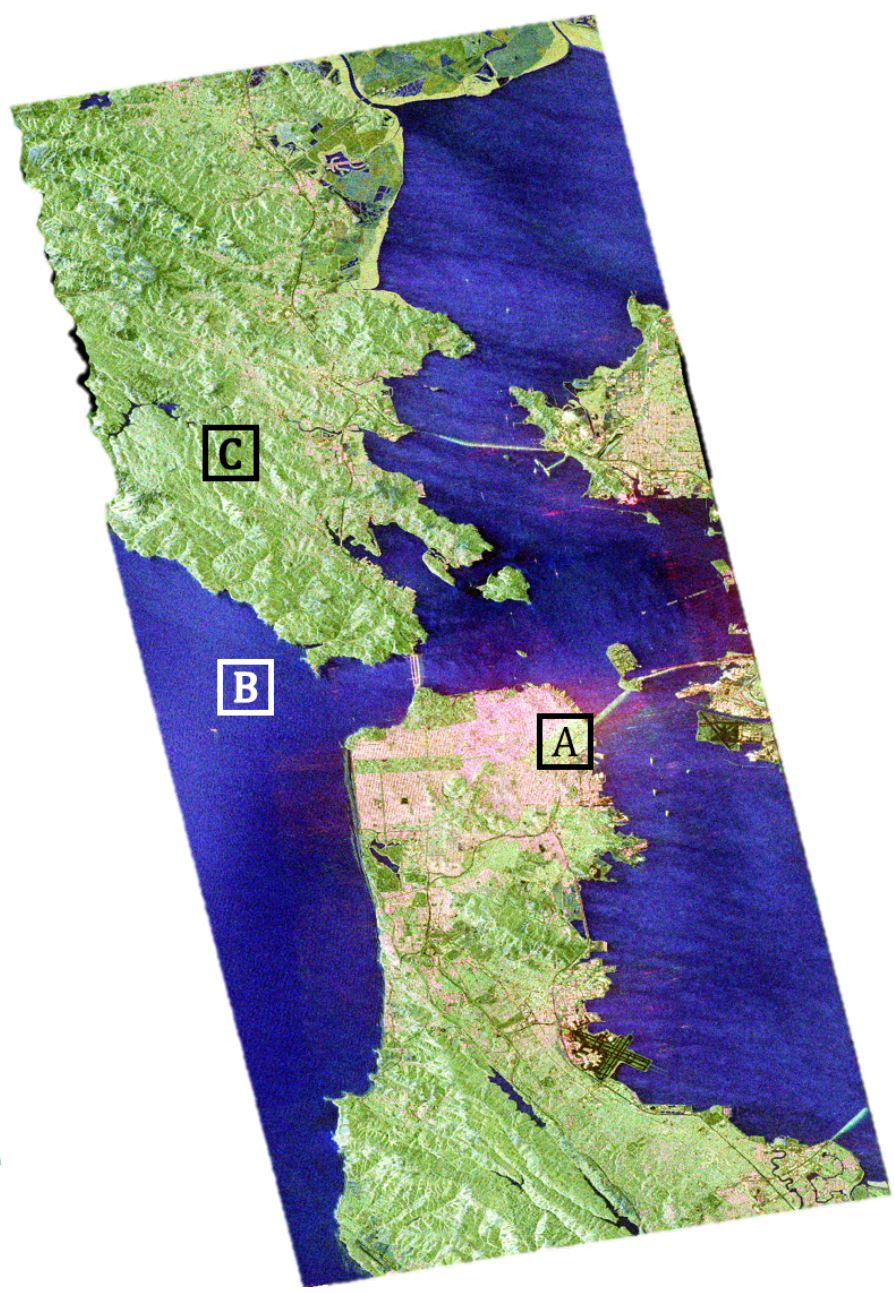

(b) Pauli RGB, San Francisco

Fig. 1. Pauli RGB images of ALOS-2 L-band (left) acquisition over Mumbai, India and RS-2 C-band (right) acquisition over San Francisco, USA.

to multiple interactions of the EM wave with distributed targets over moderately dense vegetation [33]. It can be noted that the value of $m_{\mathrm{FP}}$ is around 0.4 to 0.6 for the vegetation area. This can be attributed to the small fluctuation of $\theta_{\mathrm{FP}}$ in $\left(-4.0^{\circ}, 3.0^{\circ}\right)$ over this region as seen in Fig 4a.

For the C-band RS-2 image over San Francisco, the comparative plot between $\bar{\alpha}$ and $\theta_{\mathrm{FP}}$ in Fig. $4 \mathrm{~b}$ conveys the high discriminating power of $\theta_{\mathrm{FP}}$ over $\bar{\alpha}$. Similar to ALOS2 FP data, Fig. $4 \mathrm{~b}$ shows high values of $m_{\mathrm{FP}}$ over FSF1 and FSF3 in ocean surface. It is known that single bounce scattering dominates over the ocean surface. This is confirmed by the high odd bounce scattering $\left(\approx 45^{\circ}\right)$ in both $\theta_{\mathrm{FP}}$ and $\bar{\alpha}$. However, $\theta_{\mathrm{FP}}$ interprets a purer scattering type than $\bar{\alpha}$.

The FSF2 region constitutes an urban area that is oriented obliquely about the radar line of sight. This orientation contributes a strong cross-polarization component [34], [35] which decreases the value of $m_{\mathrm{FP}}$ over this region. However, the values of $\theta_{\mathrm{FP}}$ indicate the presence of dihedral scatterers in this region better than $\bar{\alpha}$.

It may be noted that in Fig. $4 \mathrm{~b}$ the pixels between 400 and 500 indicate high value of $m_{\mathrm{FP}}$, whereas the value of $\bar{\alpha}$ and $\theta_{\mathrm{FP}}$ are low. These low values might be due to comparable backscatter contributions from both odd and even bounce scattering mechanisms.

Hence, it is noteworthy that for both the L-and C-band SAR images, the dynamic range of $\theta_{\mathrm{FP}}$ is helpful for enhanced target characterization over $\alpha$. This improved ability might be due to the joint utilization of scattering and received wave information in $\theta_{\mathrm{FP}}$. 


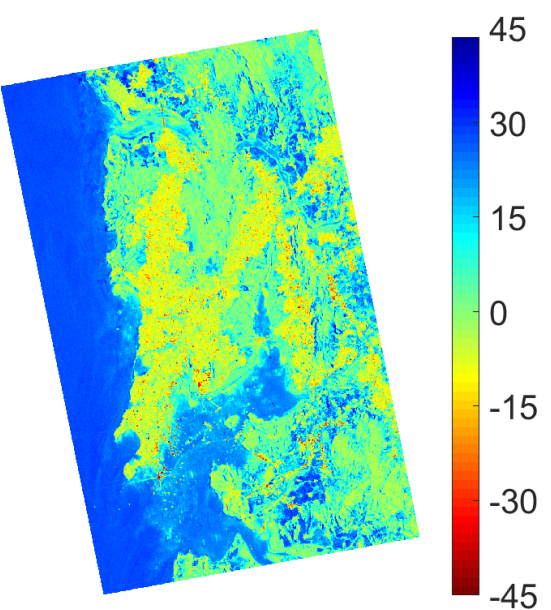

(a) $\bar{\alpha}$

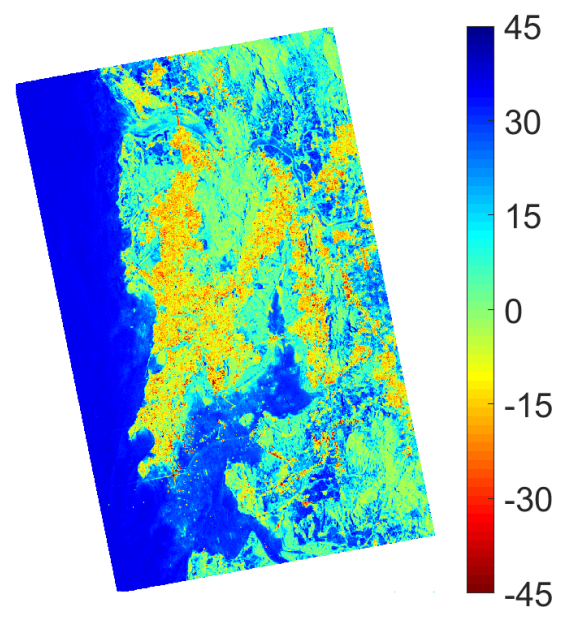

(b) $\theta_{\mathrm{FP}}$

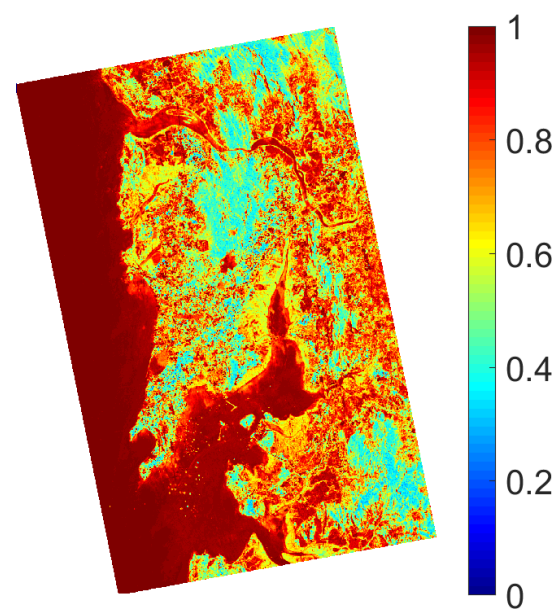

(c) $m_{\mathrm{FP}}$

Fig. 2. Images of existing $(\bar{\alpha})$ and proposed $\left(\theta_{\mathrm{FP}}\right.$ and $\left.m_{\mathrm{FP}}\right)$ parameters for ALOS-2 FP SAR data over Mumbai.

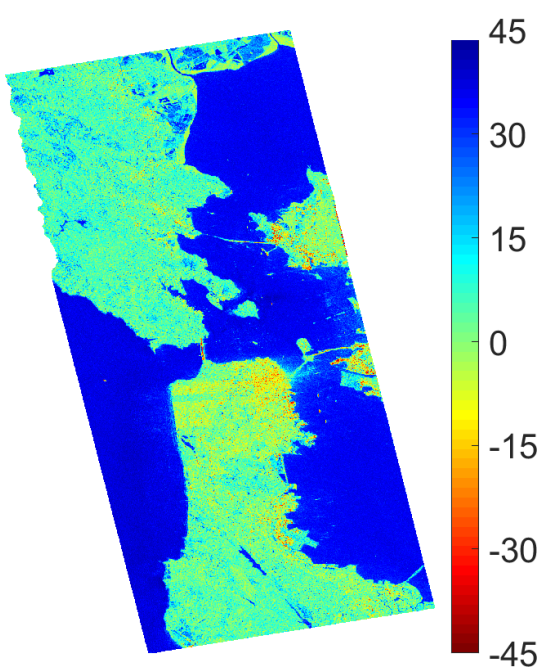

(a) $\bar{\alpha}$

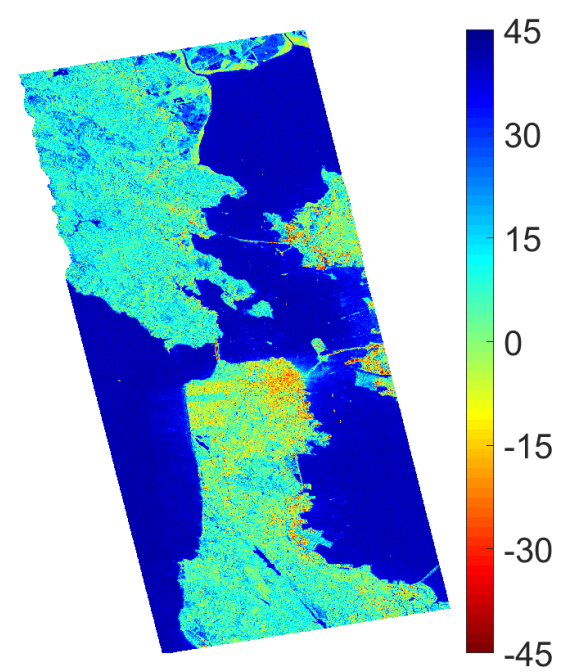

(b) $\theta_{\mathrm{FP}}$

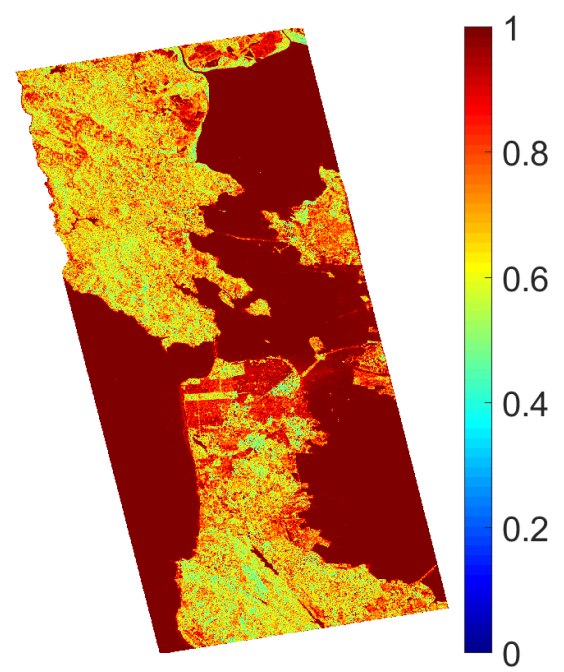

(c) $m_{\mathrm{FP}}$

Fig. 3. Images of existing $(\bar{\alpha})$ and proposed $\left(\theta_{\mathrm{FP}}\right.$ and $\left.m_{\mathrm{FP}}\right)$ parameters for RS-2 FP SAR data over San Francisco.

2) Decomposed power components: We used the proposed target scattering-type parameter $\theta_{\mathrm{FP}}$ to develop a new three-component scattering power decomposition technique. This decomposition is given in Eqs. (7), (8), and (9). Table I shows the double bounce $\left(P_{d}^{\mathrm{FP}}\right)$, volume $\left(P_{v}^{\mathrm{FP}}\right)$, and surface $\left(P_{s}^{\mathrm{FP}}\right)$ scattering power components for the (i) three-component scattering power decomposition (F3D), (ii) three-component model-based decomposition (An3D), and (iii) the proposed technique obtained with the L-band ALOS-2 Mumbai image.

The dominant surface scattering power is apparent from the three decompositions over the ocean surface (B). Here, the sample mean values of $P_{s}^{\mathrm{FP}}$ are $-12 \mathrm{~dB}$ and $-11.89 \mathrm{~dB}$ for F3D and An3D, respectively, whereas the mean value is $-11.48 \mathrm{~dB}$ for the proposed decomposition. This shows an increase of the surface scattering power by $3 \%$ to $4 \%$.

In general, an ocean surface is moderately smooth. Hence, the fraction of the volume scattering component should be minimal in the total scattering power. In this regard, the sample mean value of $P_{v}^{\mathrm{FP}}$ obtained from the proposed method is $-25.63 \mathrm{~dB}$, whereas, for both $\mathrm{F} 3 \mathrm{D}$ and An3D, the sample mean value is $\approx-20.17 \mathrm{~dB}$. Therefore, the proposed technique produces a significant and desired decrease $(\approx 5.46 \mathrm{~dB})$ in the value of the volume scattering component. 

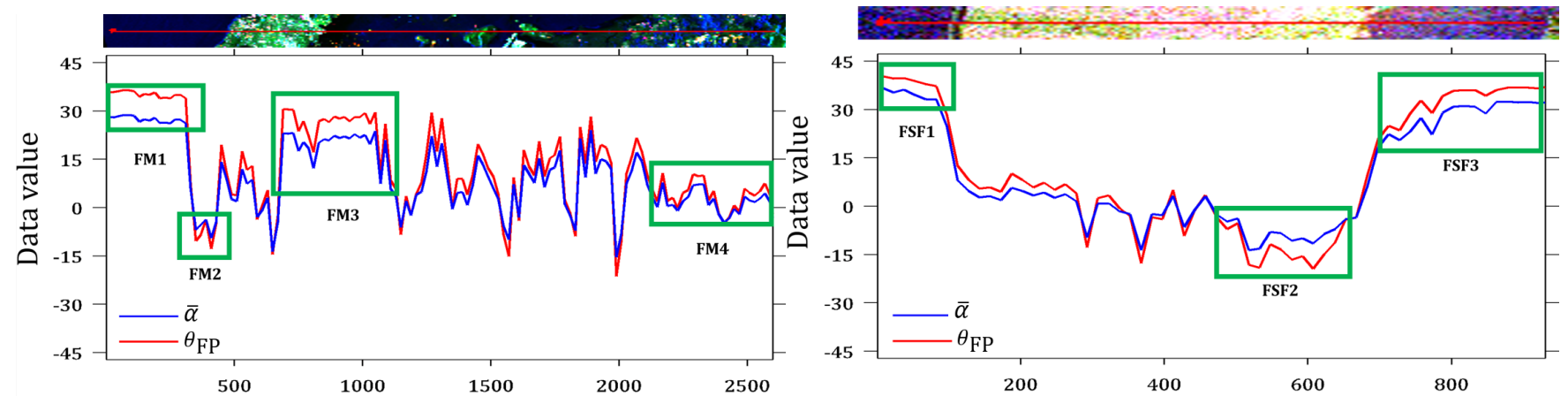

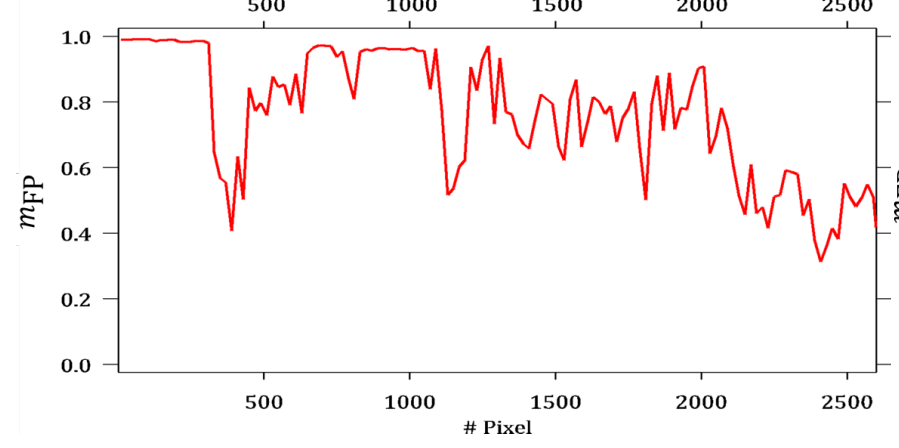

(a) L-band ALOS-2 data over Mumbai, India.

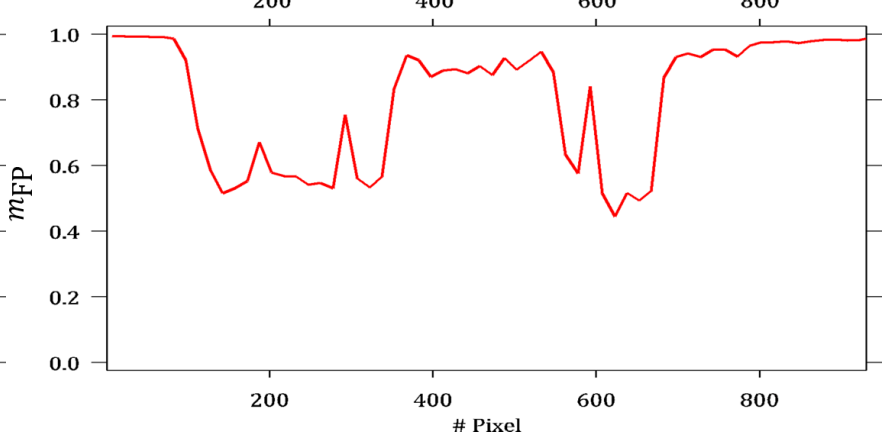

(b) C-band RS-2 data over San Francisco.

Fig. 4. Comparison of $\theta_{\mathrm{FP}}$ with $\bar{\alpha}=45^{\circ}-\alpha$, and $m_{\mathrm{FP}}$ for transects of full polarimetric data.

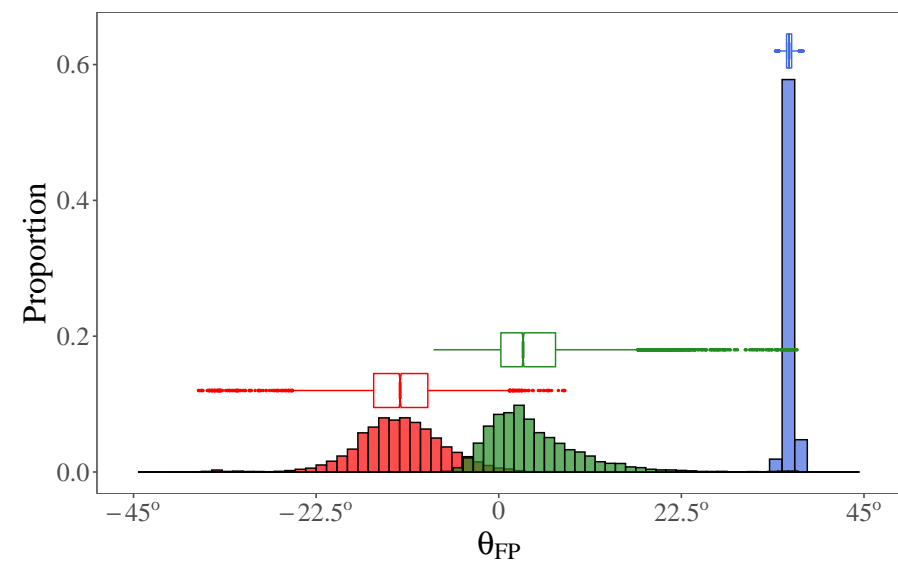

(a) ALOS-2 FP data over Mumbai: Urban (red), Sea (blue), and Forest (green)

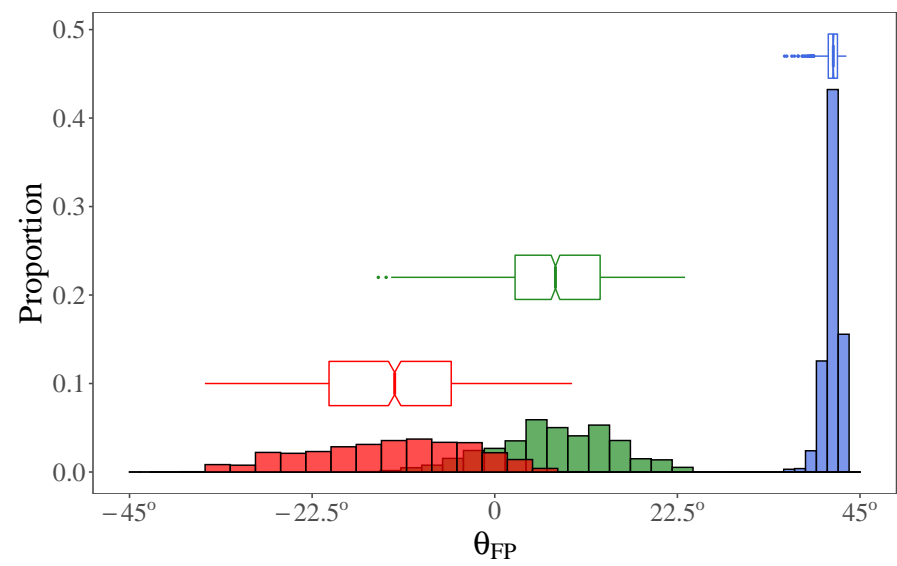

(b) Radarsat-2 FP data over San Francisco: Rotated Urban (red), Sea (blue), and Forest (green)

Fig. 5. Histograms and notched boxplots of $\theta_{\mathrm{FP}}$ over selected areas

Over the urban area (A), the sample mean value of $P_{d}^{\mathrm{FP}}$ obtained from the proposed decomposition technique is $0.27 \mathrm{~dB}$ and $0.59 \mathrm{~dB}$ higher than the $P_{d}^{\mathrm{FP}}$ obtained from An3D and F3D, respectively. On the other hand, the value of the volume scattering component, $P_{v}^{\mathrm{FP}}$ over the forest area $(\mathrm{C})$, is $0.77 \mathrm{~dB}$ and $2.1 \mathrm{~dB}$ lower than that of An3D and F3D, respectively. Also, Fig. 6 shows an increase in the double bounce scattering power over the forest area (C). A similar result from a forest area is also reported in [19]. The inclusion of the scattered wave information is useful to obtain the desired results from diverse targets.

Fig. 6 shows the results of applying the three decomposition techniques to the ALOS-2 image over Mumbai. Figures 6a, 6b, and 6c show, respectively, the images produced by F3D, An3D, and the proposed decomposition technique. Figures $6 \mathrm{~d}, 6 \mathrm{e}$, and $6 \mathrm{f}$ show the percentage of the power components over urban, ocean, and forest areas, respectively, in order to promote a qualitative analysis of these results. 
TABLE I

AVERAge DeComposition POWERS OVER DIFFERENT AREAS IN L-BAND ALOS-2 Mumbai IMAGE (IN dB SCALE)

\begin{tabular}{llccr}
\hline & Method & $P_{d}^{\mathrm{FP}}$ & $P_{v}^{\mathrm{FP}}$ & \multicolumn{1}{c}{$P_{s}^{\mathrm{FP}}$} \\
\hline \multirow{4}{*}{ Ocean area } & F3D & -19.39 & -20.17 & -12.00 \\
& An3D & -19.38 & -20.17 & -11.89 \\
& Proposed & -22.02 & -25.63 & -11.48 \\
\hline \multirow{5}{*}{ Urban area } & F3D & +2.46 & -0.44 & -2.29 \\
& An3D & +2.78 & -2.82 & -0.78 \\
& Proposed & +3.05 & -4.44 & -0.58 \\
\hline \multirow{5}{*}{ Forest area } & F3D & -18.18 & -4.50 & -15.10 \\
& An3D & -17.82 & -5.83 & -9.38 \\
& Proposed & -10.94 & -6.60 & -10.30 \\
\hline
\end{tabular}

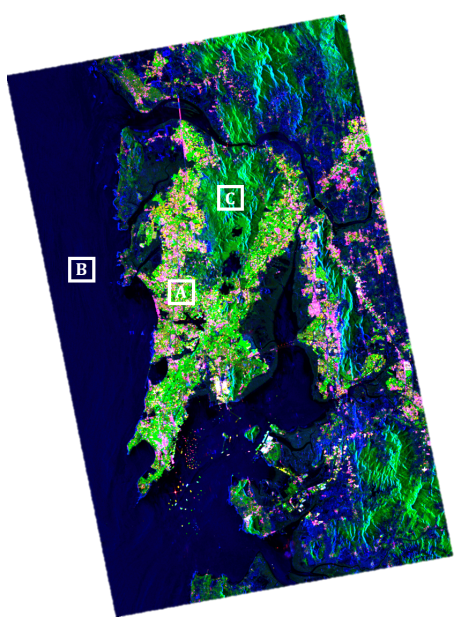

(a) $\mathrm{F} 3 \mathrm{D}$

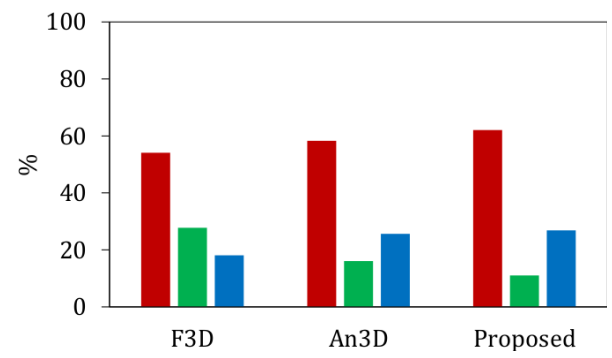

(d) Over urban area: A

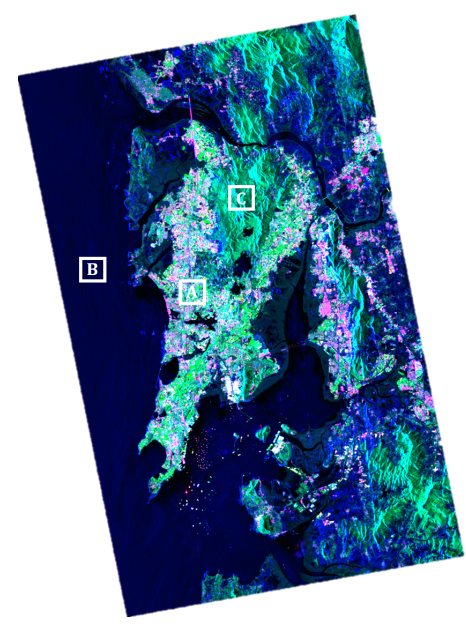

(b) An3D

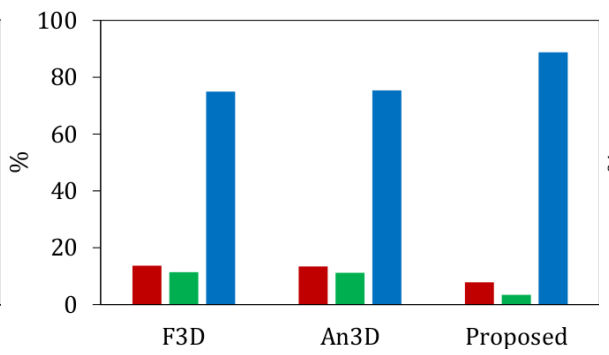

(e) Over ocean area: B

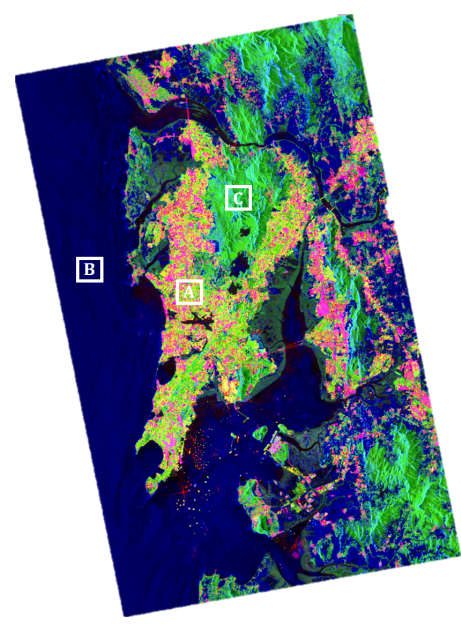

(c) Proposed

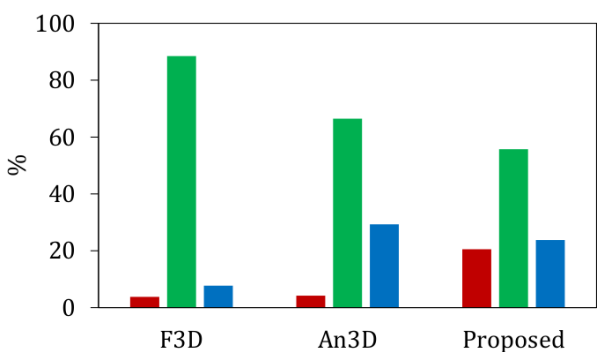

(f) Over forest area: $\mathrm{C}$

$$
P_{d}^{F P} \square P_{v}^{F P} \square P_{s}^{F P}
$$

Fig. 6. Comparison of proposed non model based decomposition powers with Freeman (F3D) and Ann (An3D) decomposition powers over different areas for full polarimetric L-band ALOS-2 SAR data over Mumbai, India.

The double bounce scattering power (in red) over the urban area (Fig. 6d) is of $54.1 \%$ according to $\mathrm{F} 3 \mathrm{D}, 58.3 \%$ according to $\mathrm{An} 3 \mathrm{D}$, and $62.1 \%$ according to the proposed technique. In contrast, the volume scattering component (in green) retrieved by F3D is $27.8 \%, 16.1 \%$ according to An3D, and $11.1 \%$ with the proposed technique.

A significant increase in the surface scattering power (in blue) over the ocean (Fig. 6e) can be noted from F3D and An3D. The surface power for the proposed technique has increased by $13.9 \%$ and $13.4 \%$ as compared to $\mathrm{F} 3 \mathrm{D}$ and $\mathrm{An} 3 \mathrm{D}$, respectively.

Over the forest area, "C", the volume scattering power $P_{v}^{\mathrm{FP}}$ has decreased $\approx 55 \%$ from $\approx 88 \%$ for F3D 
and $\approx 66 \%$ for An3D. This difference in volume scattering power might be due to the over-estimation of the model-based decomposition technique. Besides, an increase in double bounce power is also evident from the plot, which might be due to the ability of L-band EM wave to penetrate the vegetation canopy and interact with the trunks. A similar increase in double bounce power is also addressed in [19].

Table II shows the double bounce $\left(P_{d}^{\mathrm{FP}}\right)$, volume $\left(P_{v}^{\mathrm{FP}}\right)$, and surface $\left(P_{s}^{\mathrm{FP}}\right)$ scattering power components for the F3D, An3D, and the proposed technique for the C-band RS-2 San-Francisco image.

TABLE II

AVERAGE DECOMPOSITION POWERS OVER DIFFERENT AREAS IN C-BAND RS-2 SAN FRANCISCO IMAGE (IN dB SCALE)

\begin{tabular}{clccc}
\hline & Method & $P_{d}^{\mathrm{FP}}$ & $P_{v}^{\mathrm{FP}}$ & $P_{s}^{\mathrm{FP}}$ \\
\hline \multirow{3}{*}{ Ocean area } & F3D & -25.09 & -22.66 & -7.54 \\
& An3D & -25.01 & -24.00 & -7.51 \\
& Proposed & -32.29 & -33.81 & -7.36 \\
\hline \multirow{4}{*}{ Rotated urban area } & F3D & -22.84 & +4.98 & -25.32 \\
& An3D & -6.29 & +5.01 & -14.43 \\
& Proposed & +2.78 & -2.33 & -1.71 \\
\hline \multirow{2}{*}{ Forest area } & F3D & -26.74 & -6.63 & -16.94 \\
& An3D & -22.48 & -7.79 & -11.67 \\
& Proposed & -12.62 & -9.80 & -10.96 \\
\hline
\end{tabular}

Over the ocean surface (A), the dominant surface scattering power is apparent from the three decompositions. Here, the proposed technique shows marginally increased surface power by $\approx 0.15 \mathrm{~dB}$ and $\approx 0.18 \mathrm{~dB}$ as compared to the $\mathrm{An} 3 \mathrm{D}$ and $\mathrm{F} 3 \mathrm{D}$ decompositions, respectively. However, a notable increase in the double bounce scattering power over the rotated urban area (B) is observed. The double bounce scattering power for the proposed technique is $\approx 25.62 \mathrm{~dB}$ higher than $\mathrm{F} 3 \mathrm{D}$ and $\approx 9.07 \mathrm{~dB}$ higher than An3D. Although the degree of polarization over the rotated urban area ranges between 0.3 and 0.6 , the value of $\theta_{\mathrm{FP}}$ well characterizes it as even bounce scattering. This aspect can also be seen in Fig. 3 .

Over the vegetation area $(\mathrm{C})$, the sample mean of the volume scattering power, $P_{v}^{\mathrm{FP}}$, has decreased by $3.17 \mathrm{~dB}$, and $2.01 \mathrm{~dB}$ as compared to $\mathrm{F} 3 \mathrm{D}$ and $\mathrm{An3D}$, respectively. Besides, the surface and double bounce scattering powers have increased over this area. A similar result can also be seen in the ALOS-2 Mumbai image.

Fig. 7 shows the decomposed scattering power images, along with the percentage of the scattering power components. It can be noted that over the rotated urban area, both F3D and An3D show dominant volume scattering power of $99.7 \%$ and $92.1 \%$, respectively. In contrast, the proposed technique shows a dominant double-bounce scattering power of $60.1 \%$ and a volume scattering power of $18.5 \%$. Hence, over this area, the double-bounce scattering power has increased by approximately $52 \%$ to $54 \%$ with respect to both $\mathrm{F} 3 \mathrm{D}$ and $\mathrm{An} 3 \mathrm{D}$ decomposition techniques.

Over the ocean area, an increase of surface scattering power by $\approx 13 \%$ is apparent from the plots. On the other hand, as discussed earlier, the volume scattering power over the forest area has decreased by $\approx 20 \%$, and jointly the surface and double bounce scattering powers have increased by $\approx 20 \%$. Therefore, the proposed technique ascertains its ability to extract useful information about coherent targets and characterizes roll-invariant targets from fully polarimetric data.

\section{B. Compact polarimetry}

We simulated hybrid-compact polarimetric SAR data using the ALOS-2 L-band data over Mumbai and the RS-2 C-band data over San Francisco. The $2 \times 2$ covariance matrix $\mathbf{C}_{2}$ is obtained from the $3 \times 3$ covariance matrix of FP data as a function of the transmitting ellipticity $\chi$ and orientation angle $\psi$. In this study, the $\mathbf{C}_{2}$ matrix is simulated with an assumption of right circular polarization on transmit i.e., $\chi=-45^{\circ}$ and $\psi=0^{\circ}$ [36]. This particular configuration is used to simulate the compact-pol data for a 


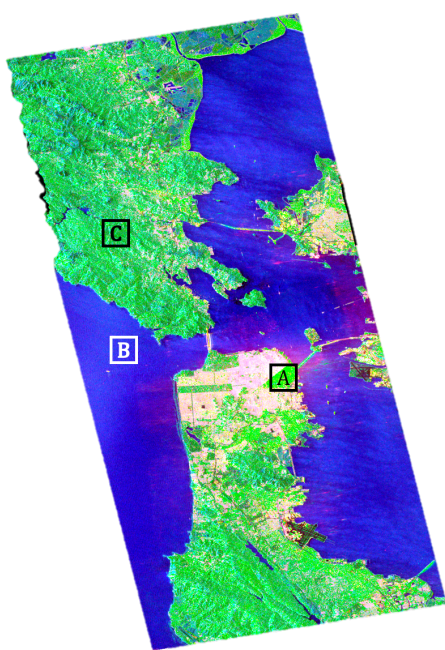

(a) $\mathrm{F} 3 \mathrm{D}$

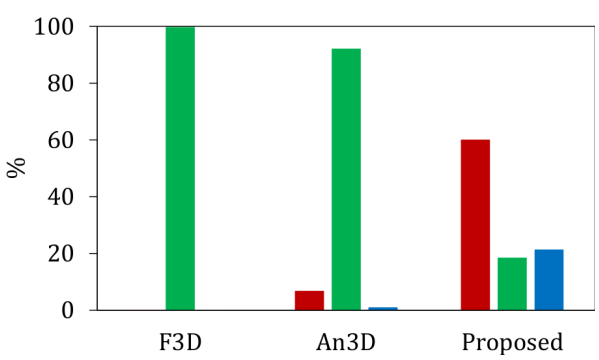

(d) Over rotated urban area: A

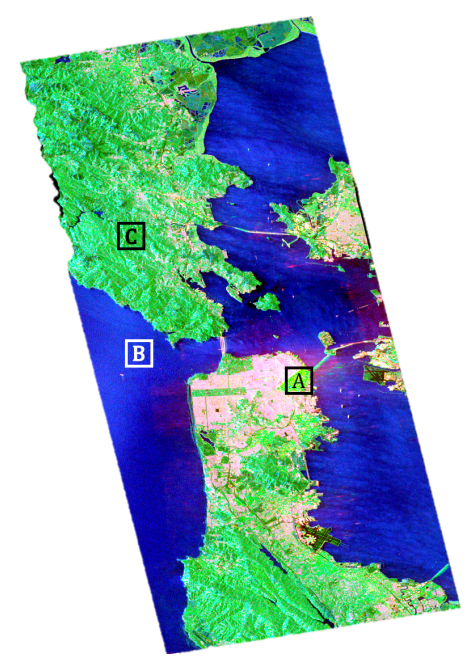

(b) An3D

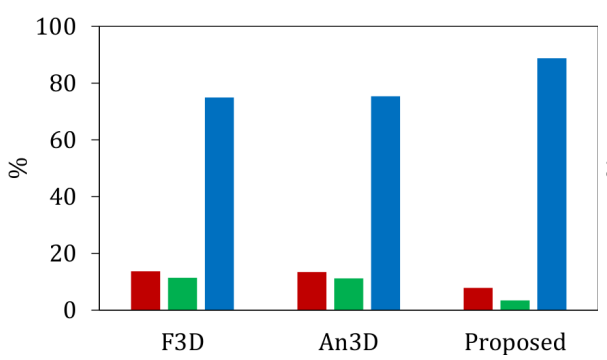

(e) Over ocean area: B

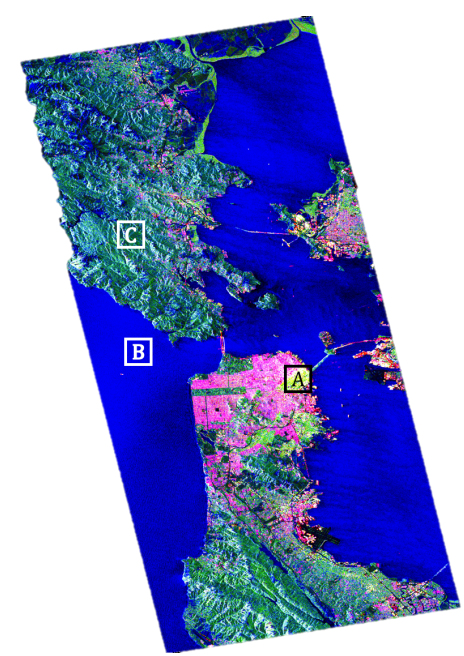

(c) Proposed

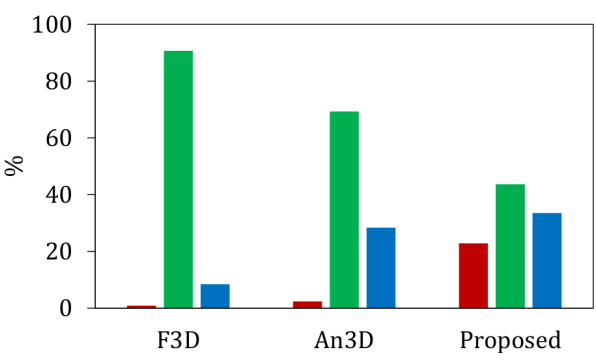

(f) Over forest area: $\mathrm{C}$

$P_{d}^{F P}-P_{v}^{F P} P_{s}^{F P}$

Fig. 7. Comparison of proposed non model based decomposition powers with Freeman (F3D) and Ann (An3D) decomposition powers over different areas for full polarimetric C-band Radarsat-2 SAR data over San Francisco, USA.

perfect circular polarization on transmit. The overall variation in $\theta_{\mathrm{CP}}, \chi$ and $m_{\mathrm{CP}}$ are shown in Fig 8 and Fig 9 which covers entire stretch from odd bounce scattering to even bounce scattering phenomenon. We then compared the target scattering type parameter, $\theta_{\mathrm{CP}}$, with the ellipticity parameter $\chi$ over different areas. This analysis is performed over the same transects used for the analysis of the FP images.

Similarly to $\theta_{\mathrm{FP}}$, the values of $\theta_{\mathrm{CP}}$ and $\chi$ varies between $-45^{\circ}$ to $45^{\circ}$. In this setting, values of $\chi$ are scaled such that both $\theta_{\mathrm{CP}}$, and $\bar{\chi}$ represent the same target within the given range. Therefore, in Figures 10a and $10 \mathrm{~b}, \bar{\chi}$ and $\theta_{\mathrm{CP}}$ indicate dihedral scattering at $-45^{\circ}$, trihedral scattering at $45^{\circ}$, and volume scattering at $0^{\circ}$.

1) Comparison of $\theta_{C P}$ with $\bar{\chi}$ : Fig. 10a shows the variation of $\theta_{\mathrm{CP}}$ and $\bar{\chi}$ over a transect in the ALOS-2 L-band image. Similar to FP, in both cases, for every 10 values over the transects, the averaged value is plotted to produce a smooth appearance. Notable differences between $\theta_{\mathrm{CP}}$ and $\bar{\chi}$ are apparent at two sections of the transect: (1) section CM1, which is over the ocean surface, and (2) section CM2, which is over a vegetation area. In section $\mathrm{CM} 1$, the value of $\bar{\chi}$ varies within $\approx 30^{\circ}$ to $32^{\circ}$ whereas, $\theta_{\mathrm{CP}}$ varies from $\approx 38^{\circ}$ to $42^{\circ}$. This variation is also apparent in Fig. 11a. Moreover, Fig. 10a shows that $m_{\mathrm{CP}}$ attains large values in CM1 section, a fact that corroborates that the scattered EM wave is majorly polarized.

Over CM2, the value of $m_{\mathrm{CP}}$ is $\approx 0$ to 0.2 , indicating a low polarization structure over the area due to random volume scattering. Hence, both $\bar{\chi}$ and $\theta_{\mathrm{CP}}$ fluctuate around $0^{\circ}$. However, the values of $\bar{\chi}$ are more erratic, between $\approx-35^{\circ}$ to $-40^{\circ}$, whereas $\theta_{\mathrm{CP}}$ fluctuates around $0^{\circ}$. Therefore, similarly to $\theta_{\mathrm{FP}}$, the dynamic range of $\theta_{\mathrm{CP}}$ is better than $\bar{\chi}$ for target characterization.

Similar results can be observed for the C-band RS-2 compact polarimetric SAR data, as shown in 


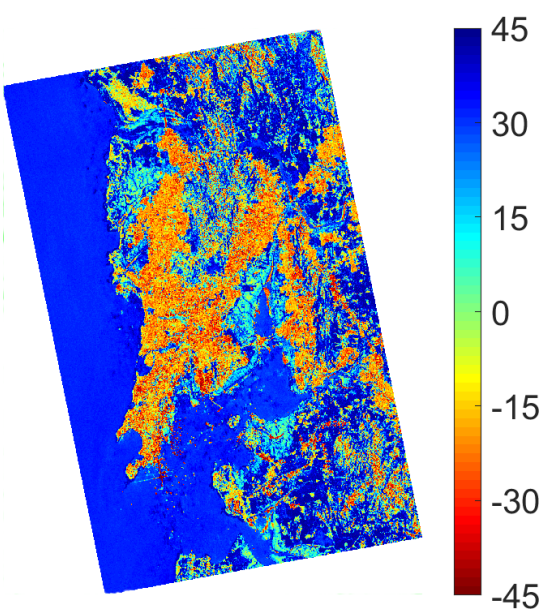

(a) $\bar{\chi}$

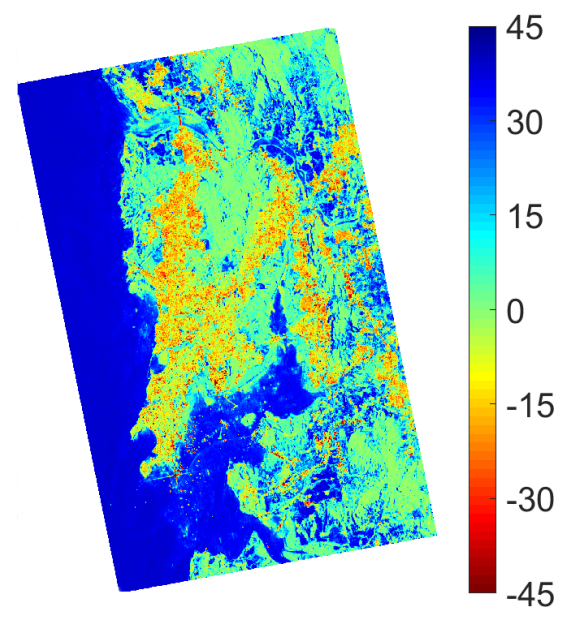

(b) $\theta_{\mathrm{CP}}$

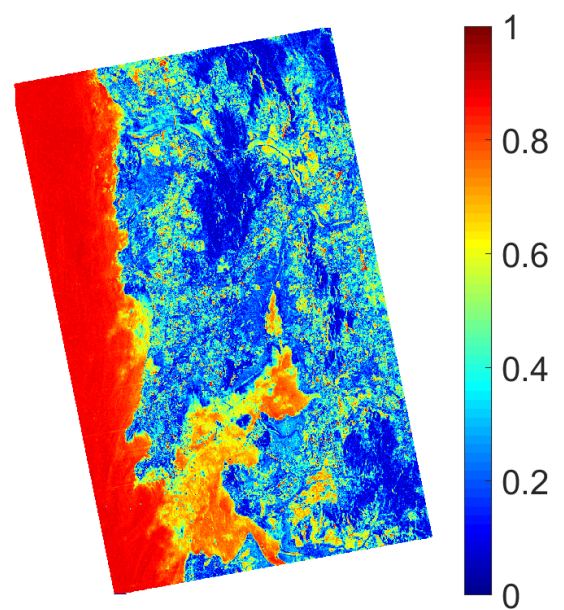

(c) $m_{\mathrm{CP}}$

Fig. 8. Visual interpretation for proposed and existing parameters for ALOS-2 CP SAR data.

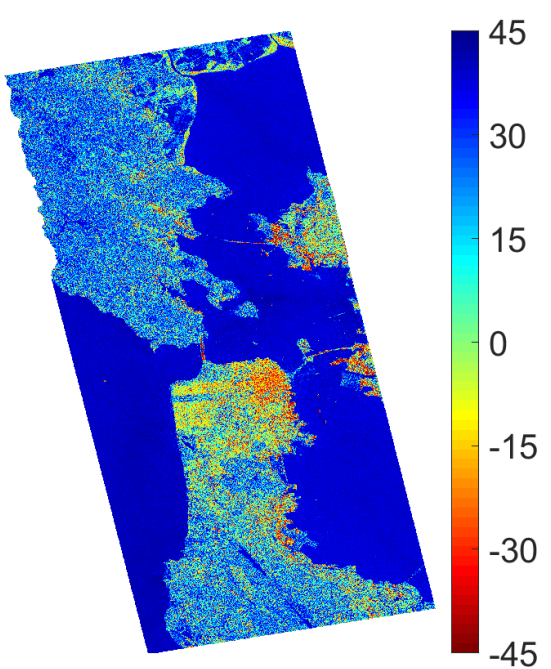

(a) $\bar{\chi}$

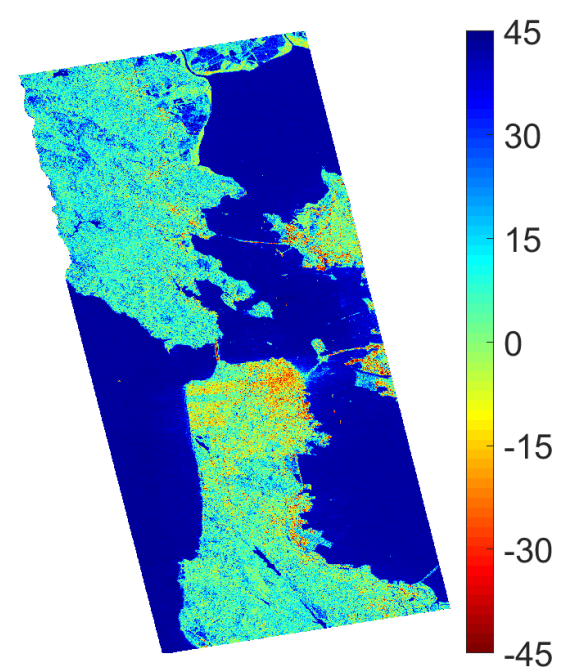

(b) $\theta_{\mathrm{CP}}$

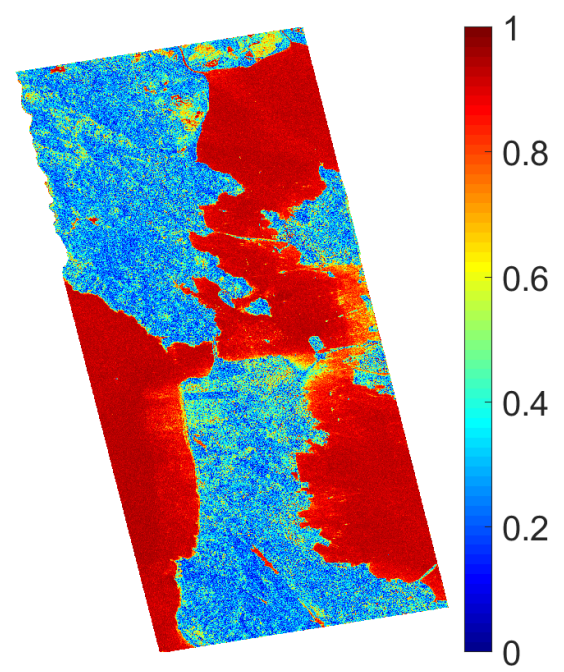

(c) $m_{\mathrm{CP}}$

Fig. 9. Visual interpretation for proposed and existing parameters for RS-2 CP SAR data.

Fig 10b. In Fig 10b, sections CSF1 and CSF3 are over the ocean surface, and section CSF2 is an area of dense vegetation. Over section CSF1 and CSF3 the value of $\theta_{\mathrm{CP}}$ is $\approx 1^{\circ}$ to $2^{\circ}$ higher than $\bar{\chi}$. Therefore, the performance of $\theta_{\mathrm{CP}}$ is marginally better than $\bar{\chi}$ for this ocean area. Furthermore, $\theta_{\mathrm{CP}}$ fluctuates around $0^{\circ}$ over the dense vegetation, whereas, the variation of $\bar{\chi}$ is inconsistent. In this case also the variation in terms of histogram can be seen in Fig. 11b.

2) Decomposed power components: Table III shows the results of the scattering power decompositions for the L-band ALOS-2 SAR data using $S-\Omega, m-\chi$, and the proposed technique. Overall, it can be noticed that the results of the proposed technique are marginally better than $S-\Omega$ and $m-\chi$. The $P_{s}^{C P}$ power is slightly better than $S-\Omega$ and $m$ - $\chi$ over the ocean. However, a significant decrease of $\approx 8.14 \mathrm{~dB}$ can be noticed in the $P_{d}^{\mathrm{CP}}$ power for the proposed technique compared to $S-\Omega$ over this region. In contrast, the difference in double bounce power between $m-\chi$, and the proposed technique is $0.31 \mathrm{~dB}$.

The value of $P_{d}^{C P}$ for both $S-\Omega$ and $m-\chi$ is $\approx 2.95 \mathrm{~dB}$ over the urban area. However, the value of $P_{d}^{\mathrm{CP}}$ for the proposed technique is $\approx 3.14 \mathrm{~dB}$. Therefore, the proposed technique discriminates better surface and double bounce scattering than $S-\Omega$ and $m-\chi$.

The value of $P_{v}^{\mathrm{CP}}$ is $\approx-7.72 \mathrm{~dB}$ for $S-\Omega$ and $\approx-7.50 \mathrm{~dB}$ for both $m-\chi$ and the proposed technique 

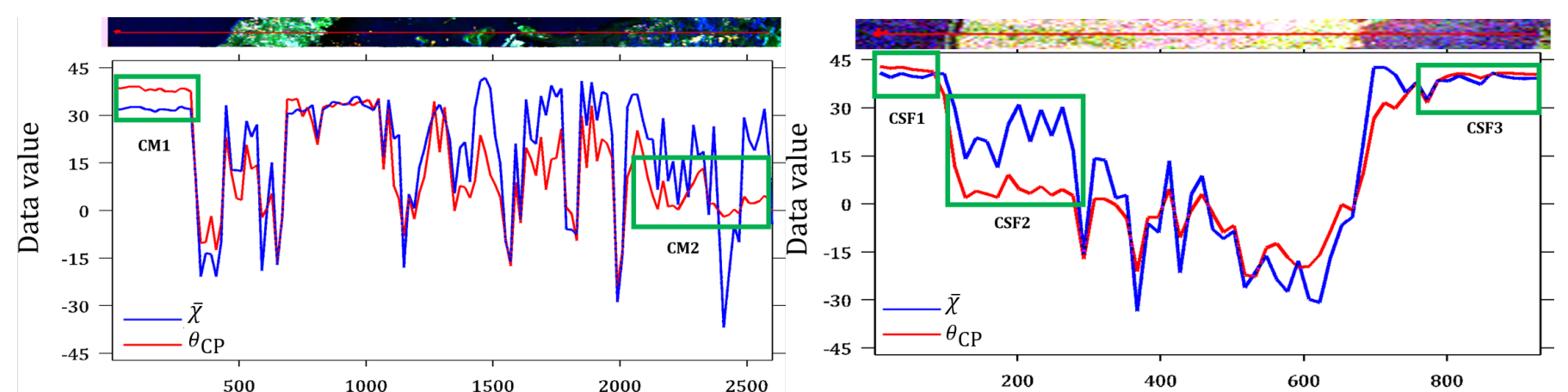

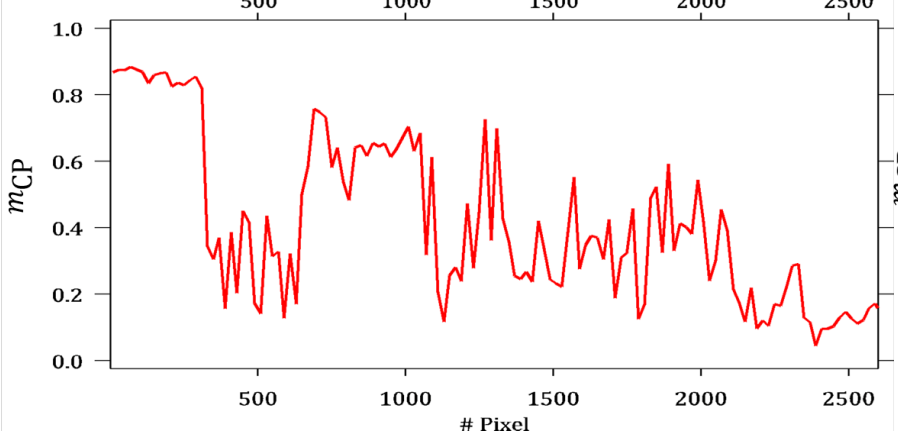

(a) L-band ALOS-2 data

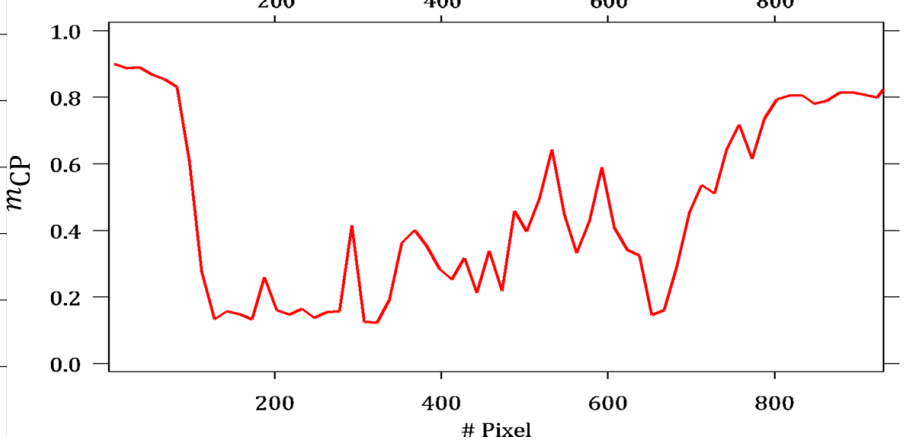

(b) C-band RS-2 data

Fig. 10. Comparison of $\theta_{\mathrm{CP}}$ with $\bar{\chi}$ for compact polarimetric data over arbitrary transects, with $\bar{\chi}=-\chi$.

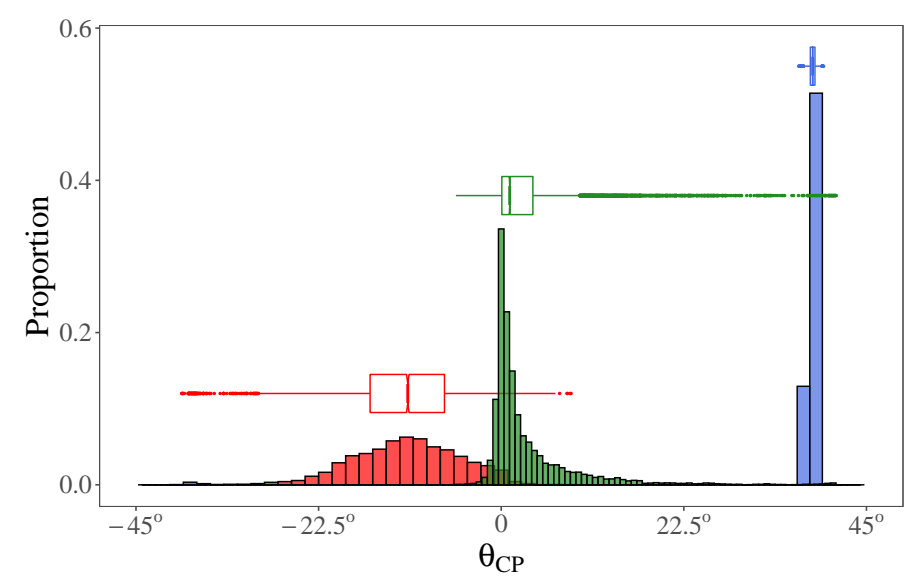

(a) ALOS-2 CP data over Mumbai: Urban (red), Sea (blue), and Forest (green)

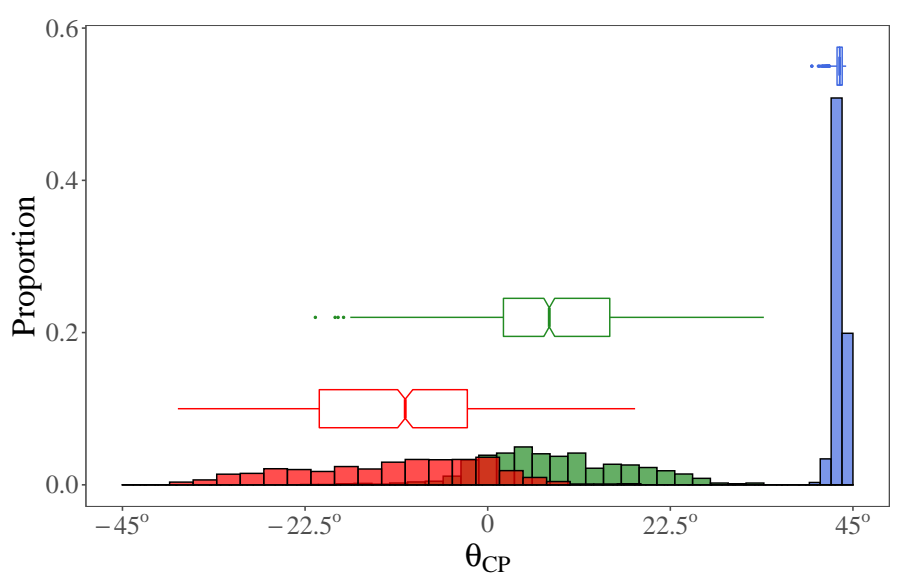

(b) Radarsat-2 CP data over San Francisco: Rotated Urban (red), Sea (blue), and Forest (green)

Fig. 11. Histograms and notched boxplots of $\theta_{\mathrm{CP}}$ over selected areas

over the forest area. It can be noted that $\Omega$ is a ratio between the polarized and the total received power, and is a function of $m_{\mathrm{CP}}$ and the transmit and receive wave properties. Due to these dependencies, the volume power component might get reduced over forests.

Moreover, it can be noted that the volume power for both $m-\chi$, and the proposed technique are the same for every target area in the scene. This is because both $m-\chi$ and the proposed technique use the depolarization fraction of the total power to compute the volume scattering power component. However, the presence of little double bounce and surface scattering power in the forest area might be due to specific structural effects and the ability of the L-band SAR wave to penetrate the forest canopy.

Similarly to the FP case, we considered three areas (A, B, and C) for comparison of the three decomposition techniques, as shown in Fig. 12. It can be noticed from the scattering power components that the dominant scattering mechanism for each area is comparable for $S-\Omega, m-\chi$, and the proposed 
TABLE III

AVERAGE DECOMPOSITION POWERS OVER DIFFERENT AREAS IN SIMULATED HYBRID-COMPACT POLARIMETRIC L-BAND ALOS-2 Mumbai image (IN dB SCALE)

\begin{tabular}{llccc}
\hline & Method & $P_{d}^{\mathrm{CP}}$ & $P_{v}^{\mathrm{CP}}$ & $P_{s}^{\mathrm{CP}}$ \\
\hline \multirow{4}{*}{ Ocean area } & $S-\Omega$ & -22.21 & -20.80 & -15.90 \\
& $m-\chi$ & -30.04 & -18.75 & -15.90 \\
& Proposed & -30.35 & -18.75 & -15.89 \\
\hline \multirow{4}{*}{ Urban area } & $S-\Omega$ & +2.95 & -1.97 & -2.43 \\
& $m-\chi$ & +2.95 & -0.36 & -5.44 \\
& Proposed & +3.14 & -0.36 & -7.06 \\
\hline \multirow{5}{*}{ Forest area } & $S-\Omega$ & -19.03 & -7.72 & -18.54 \\
& $m-\chi$ & -22.39 & -7.50 & -19.28 \\
& Proposed & -20.84 & -7.50 & -20.30 \\
\hline
\end{tabular}

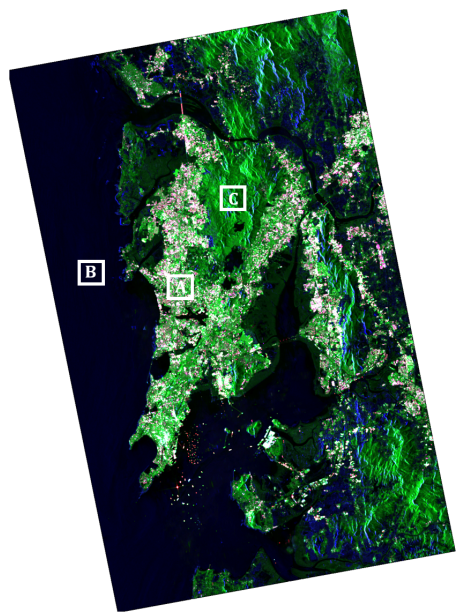

(a) $S-\Omega$

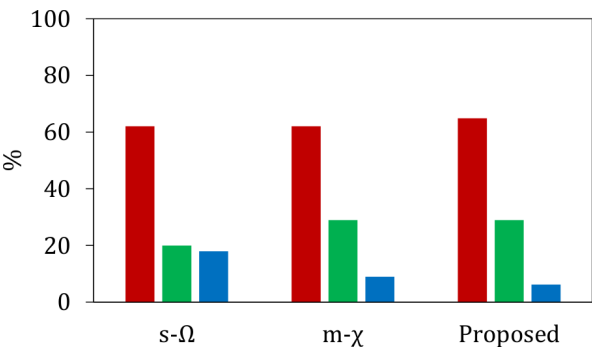

(d) Over urban area: A

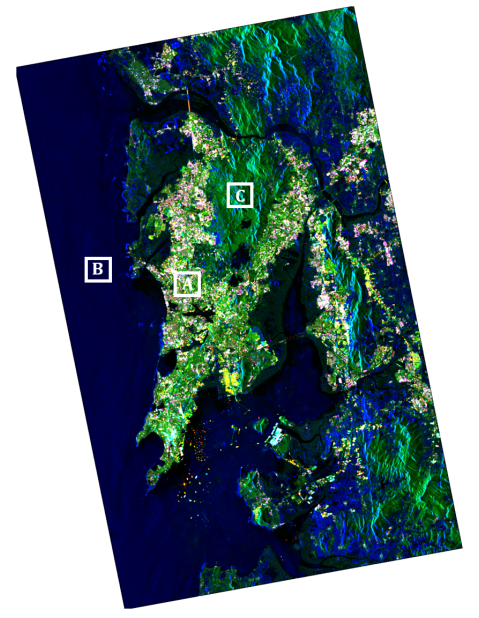

(b) $m-\chi$

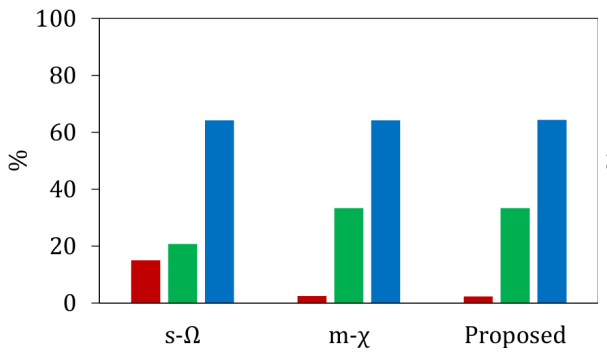

(e) Over ocean area: B

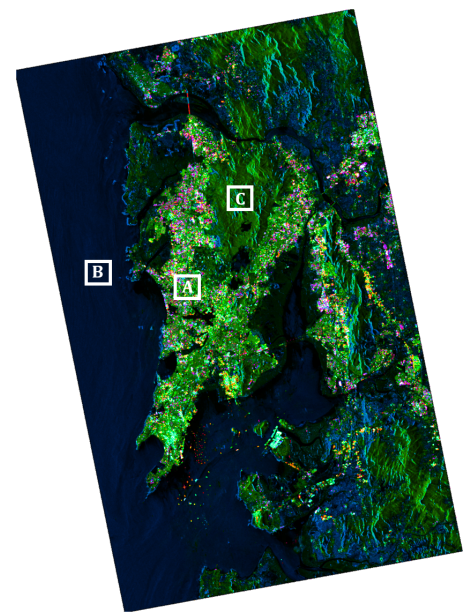

(c) Proposed

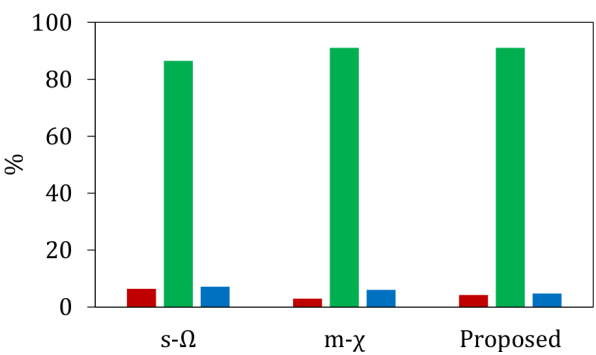

(f) Over forest area: $\mathrm{C}$

\section{$P_{d}^{C P} \longrightarrow P_{v}^{C P} P_{s}^{C P}$}

Fig. 12. Comparison of proposed non model based decomposition powers with $S-\Omega$ and $m$ - $\chi$ decomposition powers over different areas for compact polarimetric L-band ALOS-2 SAR data over Mumbai, India.

technique. Essentially, this similarity among the techniques indicates their equivalent ability to identify dominant scatterers in the scene. However, the proposed technique provides marginally better results than the other two over the urban and ocean areas for the CP SAR data.

Table IV presents a quantitative assessments of the three-component decomposition powers from $S$ $\Omega, m-\chi$, and the proposed technique using the C-band RS-2 compact polarimetric data. We used small patches over the rotated urban (A), ocean (B), and forest areas (C) for comparison. The surface scattering power over the ocean area is marginally better for the proposed technique than $S-\Omega$ and $m-\chi$. 
TABLE IV

AVERAGE DECOMPOSITION POWERS OVER DIFFERENT AREAS IN SIMULATED HYBRID-COMPACT POLARIMETRIC C-BAND RS-2 SAN FRANCISCO IMAGE (IN dB SCALE)

\begin{tabular}{clccc}
\hline & Method & $P_{d}^{\mathrm{CP}}$ & $P_{v}^{\mathrm{CP}}$ & $P_{s}^{\mathrm{CP}}$ \\
\hline \multirow{3}{*}{ Ocean area } & $S-\Omega$ & -24.79 & -25.24 & -10.66 \\
& $m-\chi$ & -32.51 & -22.40 & -10.66 \\
& Proposed & -41.74 & -22.40 & -10.63 \\
\hline \multirow{4}{*}{ Rotated urban area } & $S-\Omega$ & -0.87 & -2.80 & -4.34 \\
& $m-\chi$ & -0.88 & -1.73 & -6.52 \\
& Proposed & -0.71 & -1.73 & -7.21 \\
\hline \multirow{2}{*}{ Forest area } & $S-\Omega$ & -17.95 & -10.84 & -16.86 \\
& $m-\chi$ & -20.78 & -10.38 & -17.19 \\
& Proposed & -19.60 & -10.38 & -17.82 \\
\hline
\end{tabular}

Besides, the double bounce power is significantly lower by $\approx 16 \mathrm{~dB}$ and $\approx 9 \mathrm{~dB}$ for the proposed technique than $S-\Omega$ and $m-\chi$, respectively. This suggests that the proposed technique quantifies better the dominant scattering mechanism over the ocean surface.

Table IV shows a notable increase in double bounce scattering power over the rotated urban area by the proposed technique. The double bounce scattering power obtained from the proposed technique is $0.16 \mathrm{~dB}$ and $0.17 \mathrm{~dB}$ higher than $S-\Omega$ and $m-\chi$, respectively. However, minute $P_{v}^{\mathrm{CP}}$ power of $-1.73 \mathrm{~dB}$ from $m-\chi$ and the proposed technique is also seen over this area. The presence of this volume scattering power is likely due to the cross-polarization component of the EM wave generated by oriented urban areas about the radar line of sight [37], [38].

Over the forest area, the volume scattering power, $P_{v}^{\mathrm{CP}}$ from the proposed technique is higher than $S-\Omega$ while it is equal to that of $m-\chi$. As discussed earlier, both $m-\chi$ and the proposed technique take into account the depolarized fraction of the total power as the volume scattering component; hence, the value of this component is always identical for both techniques.

However, compared to $S-\Omega$, a $0.46 \mathrm{~dB}$ increase in the $P_{v}^{\mathrm{CP}}$ power component for the proposed technique can be noticed from the results given in Table IV. On the other hand, the $P_{s}^{\mathrm{CP}}$ power is higher than the $P_{d}^{C P}$ power over this area, which might be due to the interaction of the EM wave with the vegetation top canopy layer.

Fig. 13 shows a comparative analysis of the decomposed power images. Moreover, this figure shows the percentages of $P_{d}^{\mathrm{CP}}, P_{v}^{\mathrm{CP}}$, and $P_{s}^{\mathrm{CP}}$ over the rotated urban, ocean, and forest areas. It can be noticed that all the dominant power components are similar across all the targets. Over the rotated urban area, a $2 \%$ increase in double-bounce scattering power is noted for the proposed technique. It should also be noted that $S-\Omega, m-\chi$, and the proposed technique jointly use both scattered and received wave information. This joint utilization of the wave information aids better target characterization while improving the scattering powers.

\section{CONCLUSIONS}

We presented a novel non-model based three-component scattering power decomposition technique. In particular, this new approach combines both scattering and received wave parameters for obtaining the decomposition scattering power components.

Conversely, the classical model-based decompositions are limited to the optimization of the received covariance matrix, while the properties of the scattered wave were ignored. In this perspective, the use of the degree of polarization of the scattered wave has duly enhanced the ability to identify coherent structures within a resolution cell. This aspect is also evident from the plots of $\bar{\alpha}-\theta_{\mathrm{FP}}$ for FP data and $\bar{\chi}-\theta_{\mathrm{CP}}$ for $\mathrm{CP}$ data. 


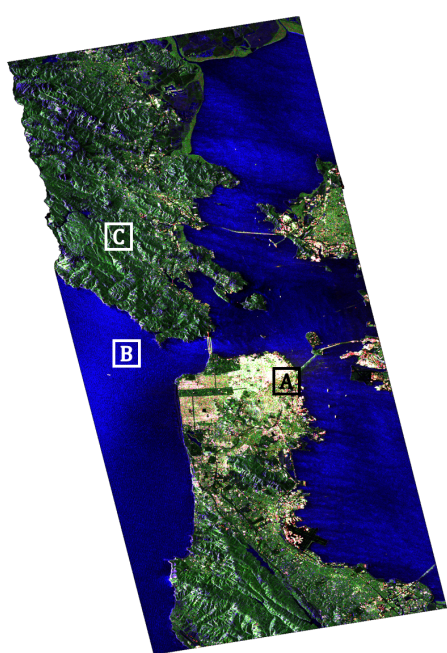

(a) $S-\Omega$

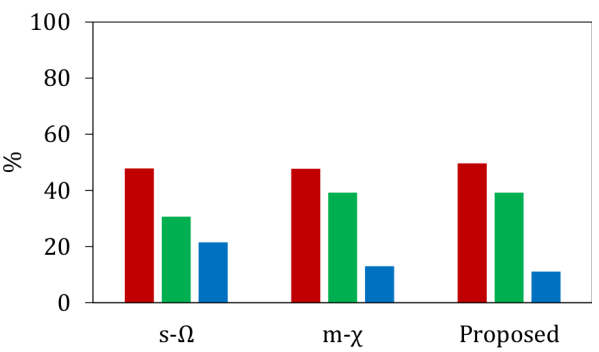

(d) Over rotated urban area: A

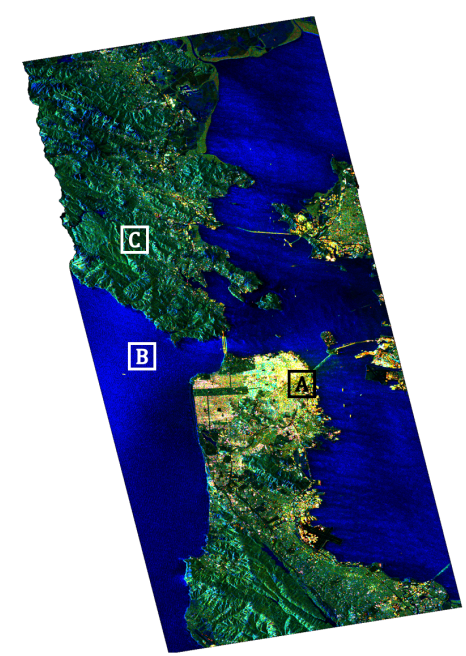

(b) $m-\chi$

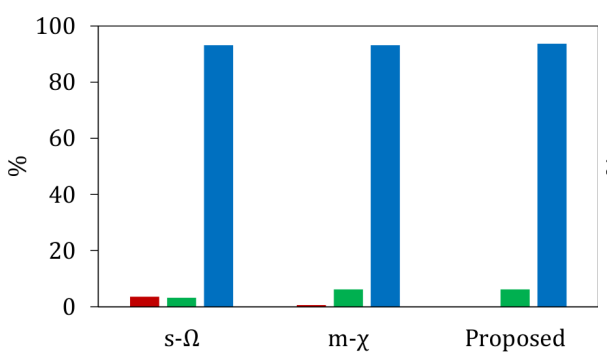

(e) Over ocean area: B

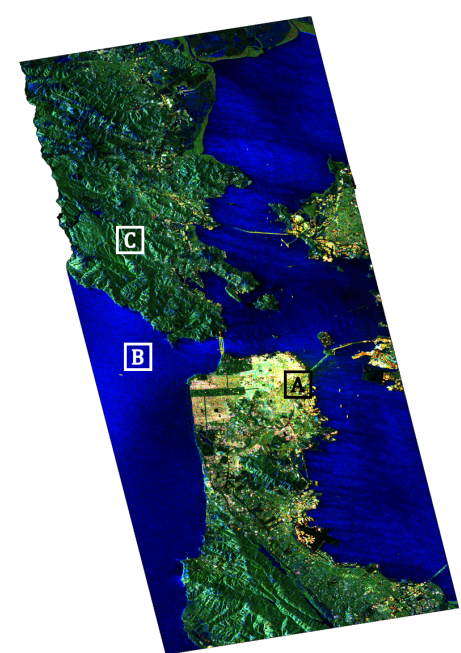

(c) Proposed

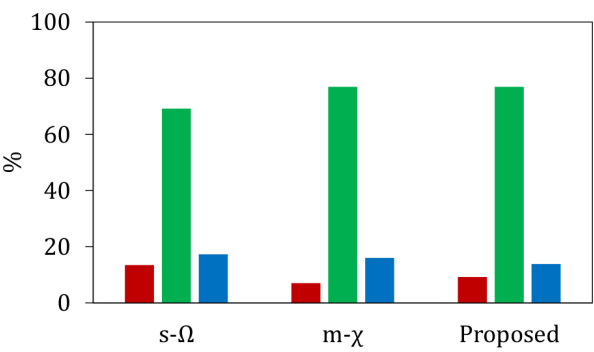

(f) Over forest area: $\mathrm{C}$

Fig. 13. Comparison of proposed non model based decomposition powers with $S-\Omega$ and $m$ - $\chi$ decomposition powers over different areas for compact polarimetric C-band RS-2 SAR data over San Francisco, USA.

Results show that our proposed technique achieves better performance than the Freeman three-component decomposition (F3D), An-Young three-component decomposition (An3D) for FP data, and $S-\Omega, m-\chi$ for $\mathrm{CP}$ data. Moreover, the proposed decomposition technique is intuitive and model-free. Hence, the overestimation of the volume power component is reduced, and the polarized power component has improved. Furthermore, the proposed technique produces non-negative power components, which is a significant drawback of model-based decompositions, as reported in several studies.

Moreover, the results also show the improvement of decomposed scattering powers over diverse regions. Specifically, as addressed earlier, the value of coherent power components has increased, which indicates the enhanced ability to extract coherent scatterers from the scene. Notably, the enhancement of coherent power estimation is evident in rotated urban areas for FP data due to the roll-invariant nature of the scattering type parameter. Indeed both F3D and An3D overestimate the volume power component over such areas.

The proposed technique clearly identifies the double bounce as the dominant power, which is also significantly higher than the volume component. Thus this proposed decomposition technique has great potential for both FP and CP SAR data.

\section{ACKNOWLEDGEMENT}

The authors would like to thank MAXAR Technologies Ltd., formerly MacDonald, Dettwiler and Associates (MDA), for providing RADARSAT-2 data and JAXA for providing the ALOS-2 PALSAR data. Also, authors acknowledge the GEO-AWS Earth Observation Cloud Credits Program, which supported 
the computation on AWS cloud platform through the project: "AWS4AgriSAR-Crop inventory mapping from SAR data on cloud computing platform."

\section{REFERENCES}

[1] S. R. Cloude and E. Pottier, "An entropy based classification scheme for land applications of polarimetric SAR," IEEE Transactions on Geoscience and Remote Sensing, vol. 35, no. 1, pp. 68-78, 1997.

[2] R. Touzi, "Target scattering decomposition in terms of roll-invariant target parameters," IEEE Transactions on Geoscience and Remote Sensing, vol. 45, no. 1, pp. 73-84, 2006.

[3] A. Freeman and S. L. Durden, "A three-component scattering model for polarimetric SAR data," IEEE Transactions on Geoscience and Remote Sensing, vol. 36, no. 3, pp. 963-973, 1998.

[4] J.-S. Lee, M. R. Grunes, T. L. Ainsworth, L.-J. Du, D. L. Schuler, and S. R. Cloude, "Unsupervised classification using polarimetric decomposition and the complex Wishart classifier," IEEE Transactions on Geoscience and Remote Sensing, vol. 37, no. 5, pp. 22492258, 1999.

[5] J.-S. Lee, M. R. Grunes, E. Pottier, and L. Ferro-Famil, "Unsupervised terrain classification preserving polarimetric scattering characteristics," IEEE Transactions on Geoscience and Remote Sensing, vol. 42, no. 4, pp. 722-731, 2004.

[6] Y. Yamaguchi, T. Moriyama, M. Ishido, and H. Yamada, "Four-component scattering model for polarimetric SAR image decomposition," IEEE Transactions on Geoscience and Remote Sensing, vol. 43, no. 8, pp. 1699-1706, 2005.

[7] M. Arii, J. J. van Zyl, and Y. Kim, "A general characterization for polarimetric scattering from vegetation canopies," IEEE Transactions on Geoscience and Remote Sensing, vol. 48, no. 9, pp. 3349-3357, 2010.

[8] M. Neumann, L. Ferro-Famil, and A. Reigber, "Estimation of forest structure, ground, and canopy layer characteristics from multibaseline polarimetric interferometric SAR data," IEEE Transactions on Geoscience and Remote Sensing, vol. 48, no. 3, pp. 1086-1104, 2009.

[9] J. J. Van Zyl, M. Arii, and Y. Kim, "Model-based decomposition of polarimetric SAR covariance matrices constrained for nonnegative eigenvalues," IEEE Transactions on Geoscience and Remote Sensing, vol. 49, no. 9, pp. 3452-3459, 2011.

[10] Y. Cui, Y. Yamaguchi, J. Yang, H. Kobayashi, S.-E. Park, and G. Singh, "On complete model-based decomposition of polarimetric SAR coherency matrix data," IEEE Transactions on Geoscience and Remote Sensing, vol. 52, no. 4, pp. 1991-2001, 2013.

[11] M. Arii, J. J. van Zyl, and Y. Kim, "Adaptive model-based decomposition of polarimetric SAR covariance matrices," IEEE Transactions on Geoscience and Remote Sensing, vol. 49, no. 3, pp. 1104-1113, 2010.

[12] J.-S. Lee and T. L. Ainsworth, "The effect of orientation angle compensation on coherency matrix and polarimetric target decompositions," IEEE Transactions on Geoscience and Remote Sensing, vol. 49, no. 1, pp. 53-64, 2010.

[13] S.-W. Chen, X.-S. Wang, S.-P. Xiao, and M. Sato, "General polarimetric model-based decomposition for coherency matrix," IEEE Transactions on Geoscience and Remote Sensing, vol. 52, no. 3, pp. 1843-1855, 2013.

[14] W. An, Y. Cui, and J. Yang, "Three-component model-based decomposition for polarimetric SAR data," IEEE Transactions on Geoscience and Remote Sensing, vol. 48, no. 6, pp. 2732-2739, 2010.

[15] Y. Yamaguchi, A. Sato, W.-M. Boerner, R. Sato, and H. Yamada, "Four-component scattering power decomposition with rotation of coherency matrix," IEEE Transactions on Geoscience and Remote Sensing, vol. 49, no. 6, pp. 2251-2258, 2011.

[16] A. Bhattacharya, G. Singh, S. Manickam, and Y. Yamaguchi, "An adaptive general four-component scattering power decomposition with unitary transformation of coherency matrix (AG4U)," IEEE Geoscience and Remote Sensing Letters, vol. 12, no. 10, pp. 2110-2114, 2015.

[17] A. Bhattacharya, A. Muhuri, S. De, S. Manickam, and A. C. Frery, "Modifying the Yamaguchi four-component decomposition scattering powers using a stochastic distance," IEEE Journal of Selected Topics in Applied Earth Observations and Remote Sensing, vol. 8, no. 7, pp. 3497-3506, July 2015.

[18] T. Eltoft and A. P. Doulgeris, "Model-based polarimetric decomposition with higher order statistics," IEEE Geoscience and Remote Sensing Letters, vol. 16, no. 6, pp. 992-996, 2019.

[19] W. An and M. Lin, "A reflection symmetry approximation of multilook polarimetric SAR data and its application to Freeman-Durden decomposition," IEEE Transactions on Geoscience and Remote Sensing, vol. 57, no. 6, pp. 3649-3660, 2019.

[20] Z. Shuang, Y. Xiangchuan, and W. Lu, "Modified version of three-component model-based decomposition for polarimetric SAR data," Journal of Systems Engineering and Electronics, vol. 30, no. 2, pp. 270-277, 2019.

[21] J.-C. Souyris, P. Imbo, R. Fjortoft, S. Mingot, and J.-S. Lee, "Compact polarimetry based on symmetry properties of geophysical media: the $\pi / 4$ mode," IEEE Transactions on Geoscience and Remote Sensing, vol. 43, no. 3, pp. 634-646, 2005.

[22] N. Stacy and M. Preiss, "Compact polarimetric analysis of X-band SAR data," EUSAR 2006, 2006.

[23] R. K. Raney, "Hybrid-polarity SAR architecture," IEEE Transactions on Geoscience and Remote Sensing, vol. 45, no. 11, pp. 3397-3404, 2007.

[24] G. G. Stokes, "On the composition and resolution of streams of polarized light from different sources," Transactions of the Cambridge Philosophical Society, vol. 9, p. 399, 1851.

[25] R. K. Raney, J. T. S. Cahill, G. W. Patterson, and D. B. J. Bussey, "The m-chi decomposition of hybrid dual-polarimetric radar data with application to lunar craters," Journal of Geophysical Research: Planets, vol. 117, no. E12, pp. n/a-n/a, 2012. [Online]. Available: http://dx.doi.org/10.1029/2011JE003986

[26] A. Bhattacharya, S. De, A. Muhuri, M. Surendar, G. Venkataraman, and A. Das, "A new compact polarimetric SAR decomposition technique," Remote sensing letters, vol. 6, no. 12, pp. 914-923, 2015.

[27] S. R. Cloude and E. Pottier, "A review of target decomposition theorems in radar polarimetry," IEEE Transactions on Geoscience and Remote Sensing, vol. 34, no. 2, pp. 498-518, 1996.

[28] A. Swartz, H. Yueh, J. Kong, L. Novak, and R. Shin, “Optimal polarizations for achieving maximum contrast in radar images,” Journal of Geophysical Research: Solid Earth, vol. 93, no. B12, pp. 15 252-15 260, 1988. 
[29] R. Touzi, K. Omari, B. Sleep, and X. Jiao, "Scattered and received wave polarization optimization for enhanced peatland classification and fire damage assessment using polarimetric PALSAR," IEEE Journal of Selected Topics in Applied Earth Observations and Remote Sensing, vol. 11, no. 11, pp. 4452-4477, 2018.

[30] R. Barakat, "Degree of polarization and the principal idempotents of the coherency matrix," Optics Communications, vol. 23, no. 2, pp. 147-150, 1977.

[31] S. Cloude and E. Pottier, "An entropy based classification scheme for land applications of polarimetric SAR," IEEE Transactions on Geoscience and Remote Sensing, vol. 35, no. 1, pp. 68-78, 1997.

[32] D. J. McLaughlin, N. Allan, E. M. Twarog, and D. Trizna, "High resolution polarimetric radar scattering measurements of low grazing angle sea clutter," IEEE Journal of Oceanic Engineering, vol. 20, no. 3, pp. 166-178, 1995.

[33] I. Brown, S. Mwansasu, and L.-O. Westerberg, "L-band polarimetric target decomposition of mangroves of the Rufiji Delta, Tanzania," Remote Sensing, vol. 8, no. 2, p. 140, 2016.

[34] S.-H. Hong and S. Wdowinski, "Double-bounce component in cross-polarimetric SAR from a new scattering target decomposition," IEEE Transactions on Geoscience and Remote Sensing, vol. 52, no. 6, pp. 3039-3051, 2013.

[35] T. Ainsworth, D. Schuler, and J.-S. Lee, "Polarimetric SAR characterization of man-made structures in urban areas using normalized circular-pol correlation coefficients," Remote Sensing of Environment, vol. 112, no. 6, pp. 2876-2885, 2008.

[36] R. Sabry and P. Vachon, "A unified framework for general compact and quad polarimetric SAR data and imagery analysis," Geoscience and Remote Sensing, IEEE Transactions on, vol. 52, no. 1, pp. 582-602, Jan 2014.

[37] R. Guinvarc'h and L. Thirion-Lefevre, "Cross-polarization amplitudes of obliquely orientated buildings with application to urban areas," IEEE Geoscience and Remote Sensing Letters, vol. 14, no. 11, pp. 1913-1917, 2017.

[38] D. K. Atwood and L. Thirion-Lefevre, "Polarimetric phase and implications for urban classification," IEEE Transactions on Geoscience and Remote Sensing, vol. 56, no. 3, pp. 1278-1289, 2017. 